Postprint: Tijskens A., Roels S., Janssen H., 2019. Hygrothermal assessment of timber frame walls using a convolutional neural network. Building and Environment 193, 107652

https://doi.org/10.1016/j.buildenv.2021.107652

\title{
HYGROTHERMAL ASSESSMENT OF TIMBER FRAME WALLS USING A CONVOLUTIONAL NEURAL NETWORK
}

\author{
Astrid Tijskens ${ }^{a, *}$, Staf Roels ${ }^{a}$, Hans Janssen ${ }^{a}$ \\ ${ }^{a}$ KU Leuven, Department of Civil Engineering, Building Physics and Sustainable Design, Kasteelpark Arenberg 40 Bus 2447,3001 Heverlee, \\ Belgium \\ * Corresponding author. E-mail address: astrid.tijskens@kuleuven.be; ORCID ID: https://orcid.org/0000-0003-4568-7769
}

\section{AbSTRact}

A correct design of a timbre frame wall's composition is vital to avoid moisture damage. Unfortunately, currently, no general guidelines exist to determine the most optimal wall composition in a specific context. To develop such general guidelines, a comprehensive study is required, taking into account the inherent uncertainty and variability of involved input parameters. Such a probabilistic assessment is typically carried out through a Monte-Carlo approach, which easily becomes computationally inhibitive. This paper thus makes use of a metamodel, which mimics the complex hygrothermal model while being considerably faster. The authors previously developed a convolutional neural network and demonstrated its' capacity to predict the highly non-linear hygrothermal response of a massive masonry wall. In this paper, this network is adapted to predict the hygrothermal response for timber frame walls. A hyper-parameter optimisation is performed, leading to rules-of-thumb on the network architecture. It is shown that the network can accurately predict the hygrothermal time series, and that it can be employed with confidence to estimate the moisture damage risks. Subsequently, the network is used to calculate the hygrothermal response of 96 timber frame wall types, taking into account all influencing uncertainties. The results indicated that timber frame wall compositions should not be recommend based solely on the $s_{d}$-ratio between vapour and wind barrier. A lower limit for the $s_{d}$-ratio appears a good criterion to avoid mould growth, if adapted to the climate and cladding type. To avoid condensation, one should ensure either the insulation or the wind barrier can buffer the excess moisture. 
Postprint: Tijskens A., Roels S., Janssen H., 2019. Hygrothermal assessment of timber frame walls using a convolutional neural network. Building and Environment 193, 107652

https://doi.org/10.1016/j.buildenv.2021.107652

\section{KEYWORDS}

Metamodelling; Convolutional neural networks, Time series modelling; Probabilistic assessment; Hygrothermal assessment; Timber frame walls

\section{Introduction}

In recent years, there is a growing market share of timber frame constructions across Europe, due to its suitability for low-energy buildings. A major disadvantage of timber frame construction, however, is its susceptibility to moisture damage. According to Straube and Finch [1], vapour transfer through the wall by diffusion and/or advection is one of the most common moisture sources leading to deterioration of timber frame constructions. This can be avoided by a correct design of the building component's composition, so that high relative humidity and interstitial condensation are prevented. In Europe, timber frame walls typically consist of a wind barrier at the cold exterior side and a vapour barrier/retarder at the warm interior side. In cold climates, the vapour barrier must have a higher vapour resistance than the wind barrier, to ensure vapour that entered the construction at the inside can dry out towards the outside [2]. Many different wind and vapour barrier materials exist, with varying characteristics. Yet, there are no general guidelines available as to which combinations of wind and vapour barrier are safe in a specific context. The Belgium Building Research Institute proposed a rule of thumb which advises the ratio between the vapour resistances of vapour and wind barrier to be at least 6 but preferably 15 in case of timber frame walls with a brick veneer [2]. In Finland, a ratio of 5 is recommended for timber frame walls with wooden sidings [3]. For other European countries or climates, no such rules have been proposed to the authors' knowledge. Furthermore, these rules do not take into account the moisture buffering capacity of the structure nor specific climatic aspects, and hence do not guarantee an optimal solution. One could evaluate the hygrothermal performance of a specific wall construction for a specific project, though in practice this is rarely done as it is too time consuming, especially when not all variables are known or multiple options need to be assessed. A study by the Oak Ridge National Laboratory performed an in-depth analysis of the hygrothermal performance of timber frame walls [4]. However, only 30 different timber frame wall compositions 
Postprint: Tijskens A., Roels S., Janssen H., 2019. Hygrothermal assessment of timber frame walls using a convolutional neural network. Building and Environment 193, 107652

https://doi.org/10.1016/j.buildenv.2021.107652

were included and no variability or uncertainty in boundary conditions were considered, most likely due to computational time constraints.

To come to general guidelines, valid for different climates and various timber frame wall compositions, a comprehensive study is required, taking into account the inherent uncertainty and variability of involved input parameters. Such a probabilistic assessment is typically carried out through a MonteCarlo approach [5], where the uncertain input parameters' distributions are sampled several times and a deterministic simulation is executed for each sampled parameter combination. Unfortunately, this approach would be too time-expensive here due to the high amount of required simulations. Therefore, the hygrothermal model can be replaced by a metamodel, which mimics the complex hygrothermal model while being considerably faster. A metamodel is typically trained on a small dataset calculated with the original model, to learn the patterns between input and output. After this training, the metamodel can be used to calculate the hygrothermal response of any input combination in the range it was trained on. Such a metamodel would thus allow to quickly determine adequate combinations of wind and vapour barrier under given conditions, hence eliminating the computational time constraint. The objective of the current study is to develop a fast and accurate metamodel that allows performing a comprehensive probabilistic assessment of timber frame constructions, required to come to general guidelines, valid for different climates and various timber frame wall compositions.

The authors showed previously that a convolutional neural network for time series is highly adequate for such purpose [6]-[8] as it is able to capture the complex time-dependent patterns of the hygrothermal response and allows flexibility in the desired post-processing. Hence, a similar network architecture as in [6] is used in this study to replace the hygrothermal model used to evaluate the hygrothermal performance of timber frame walls.

The next section describes the different types of timber frame walls that are included in this study, and specifies the probabilistic set-up. Subsequently, section 3 covers the convolutional neural network architecture, the hyper-parameter optimisation and the network development. Finally, in section 4, 
Postprint: Tijskens A., Roels S., Janssen H., 2019. Hygrothermal assessment of timber frame walls using a convolutional neural network. Building and Environment 193, 107652

https://doi.org/10.1016/j.buildenv.2021.107652

the developed network is employed to evaluate the hygrothermal performance of the timber frame walls and to derive general rules for the applicability of wind and vapour barrier combinations.

\section{Hygrothermal simulation object}

The calculation object in this study is a 1D cross section of a timber frame wall, as shown in Figure 1.

Different cladding materials, insulation types, wind barriers and vapour barriers are considered, as shown in Table 1. All possible combinations of these options are considered, thus resulting in 96 different wall types. Note that practical requirements, such as the use of a building board for the wind or vapour barrier to ensure sufficient structural stiffness, are not considered here, in order to attain a wide range of combinations of vapour and wind barrier properties. Furthermore, rainwater leakage into the wall structure is not considered in this study. The used material characteristics are shown in Figure 2 and Figure 3. The Celit and Duripanel material characteristics were measured [9], all other material data comes from the Delphin 6.0 material database [10].
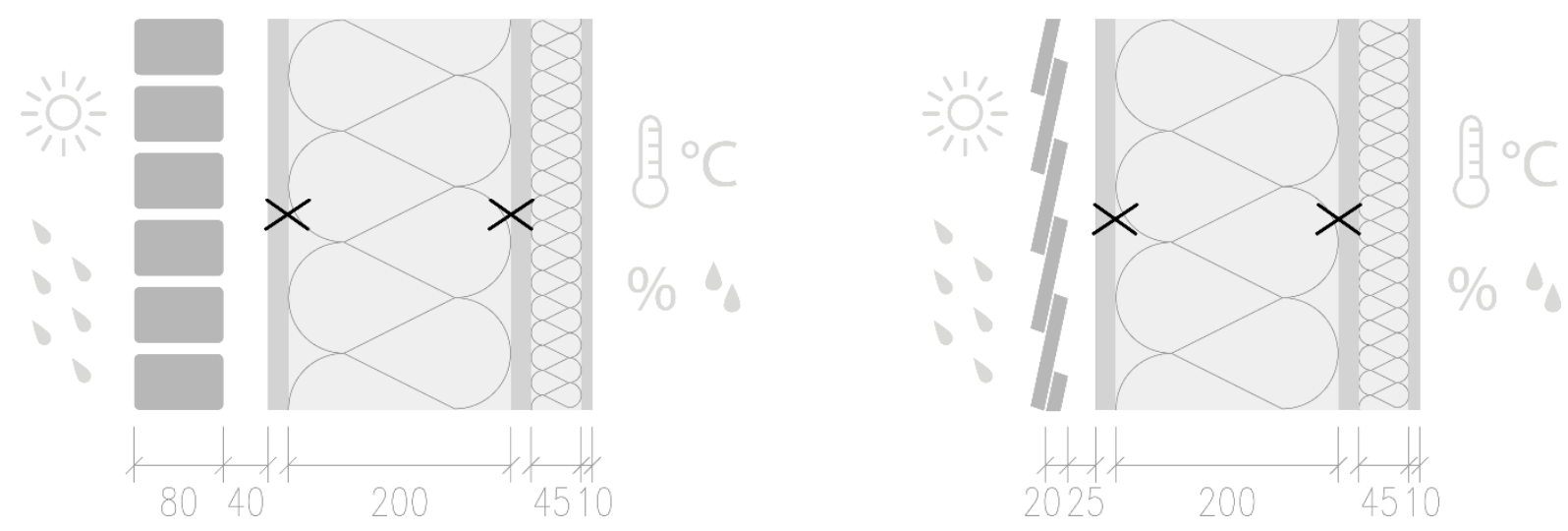

Figure 1: Schematic representation of the timber frame wall with brick veneer (left) and sidings (right), with indication of the positions considered for the hygrothermal evaluation. All dimensions are given in $\mathrm{mm}$.

Table 1: The different options for the timber frame wall construction

\begin{tabular}{llll}
\hline Façade cladding & Wind barrier & Insulation type & Vapour barrier \\
\hline Brick veneer $80 \mathrm{~mm}+$ cavity $40 \mathrm{~mm}$ & Celit $^{1} 18 \mathrm{~mm}$ & Cellulose & Climajet SD vario \\
Wooden sidings $20 \mathrm{~mm}+$ cavity $25 \mathrm{~mm}$ & Duripanel $^{2} 18 \mathrm{~mm}$ & Mineral wool & OSB $18 \mathrm{~mm}$ \\
& OSB $18 \mathrm{~mm}$ & & PE foil 1 \\
& PE foil 1 & PE foil 5
\end{tabular}


Postprint: Tijskens A., Roels S., Janssen H., 2019. Hygrothermal assessment of timber frame walls using a convolutional neural network. Building and Environment 193, 107652

https://doi.org/10.1016/j.buildenv.2021.107652

PE foil 5

Dupont Tyvek DPT
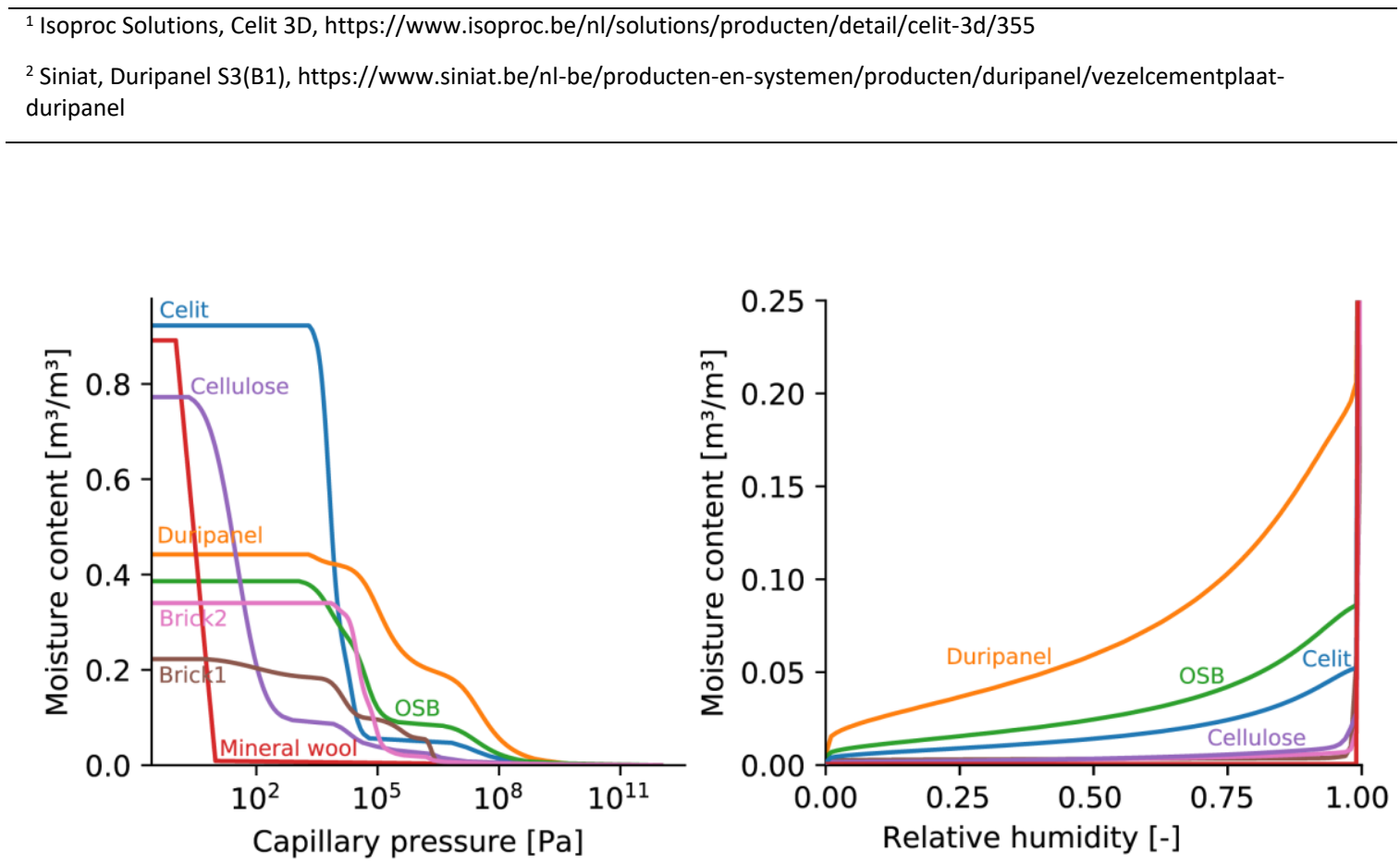

Figure 2: Material moisture capacity properties.
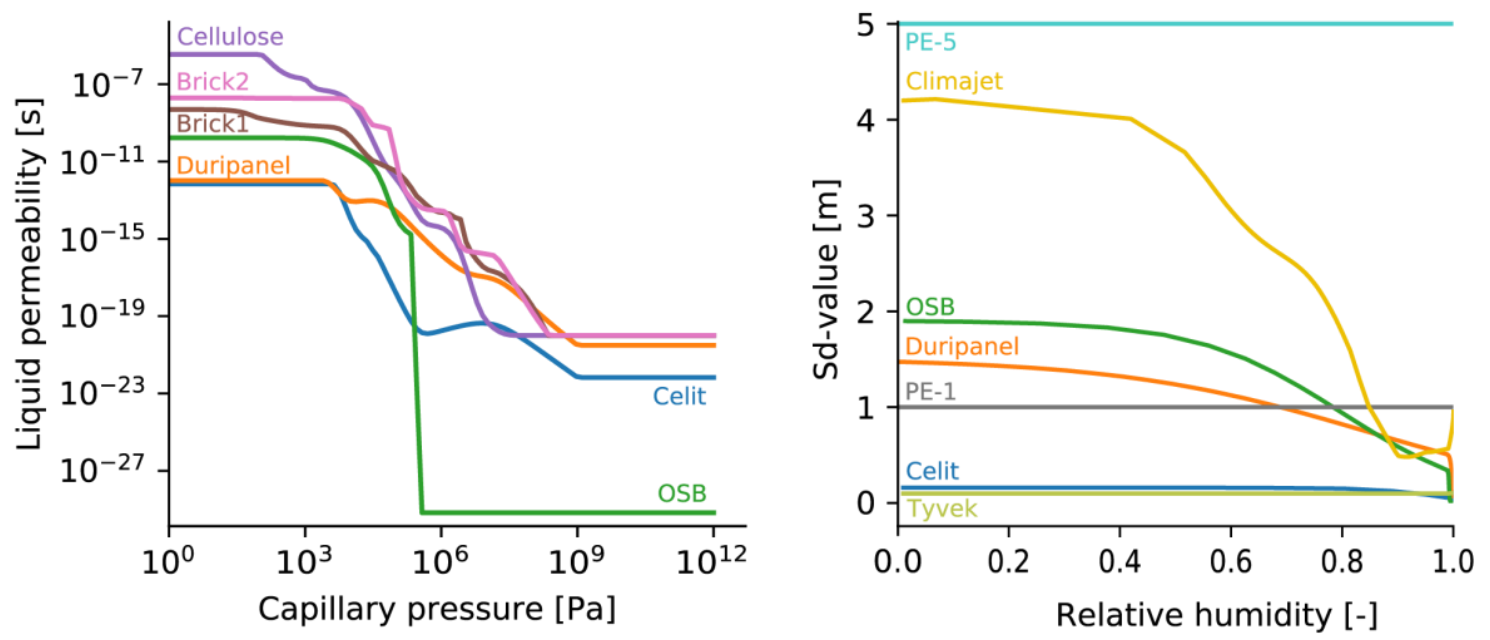

Figure 3: Material moisture transport properties.

All characteristics and boundary conditions that are expected to significantly influence the hygrothermal performance of the 1D timber frame walls are considered probabilistic; these input variables' values are show in Table 2. The interior climate is calculated according to EN 15026 [11] and variability in building use is included by using two different humidity loads A and B. Variability in exterior climatic conditions is included by using different years of climate data of 54 European 
Postprint: Tijskens A., Roels S., Janssen H., 2019. Hygrothermal assessment of timber frame walls using a convolutional neural network. Building and Environment 193, 107652

https://doi.org/10.1016/j.buildenv.2021.107652

locations of ClimateforCulture [12]. Only climates belonging to the Köppen-Geiger climate classes [13]

$\mathrm{Cfb}, \mathrm{Cfc}, \mathrm{Dfb}$ and Dfc are used, since timber frame wall are most commonly used in these climates.

Climate data of the period 2020-2050 is used, which takes into account the A1B climate change scenario [14]. Via uniform distributions of the wall orientation, solar absorption and exposure to winddriven rain, variability in the wall conditions is considered as well. The wind-driven rain load is calculated by using the catch ratio as described in [15]. The catch ratio relates the wind-driven rain (WDR) intensity on a facade to the unobstructed horizontal rainfall intensity and is a function of the reference wind speed and the horizontal rainfall intensity for a given position on the building facade and wind direction. In this model, the exposure factor covers the variability in wall position and potential shelters, trees or surrounding buildings. Additionally, the variation of the wind speed is taken into account in the convective heat transfer coefficient, via equation (1) (EN ISO 06946) where $h_{0}=$ $4 \mathrm{~W} / \mathrm{m}^{2} \mathrm{~K}$ and $k_{e}=1$.

$$
h_{c}=h_{0}+k_{s} \cdot v_{\text {wind }}^{k_{e}}
$$

The exterior moisture transfer coefficient is related to the exterior heat transfer coefficient through the Lewis relation. Previous studies [9], [16], [17] showed that the air change rate of the cavity is significantly different for the walls with brick veneer or wooden sidings. Based on the measurements of [16], a different uniform distribution is considered for both cladding types: in case of a brick façade, the cavity air change rate is assumed to be uniformly distributed between 2 and $201 / \mathrm{h}$, while in case of wooden sidings, a uniform distribution between 50 and $5001 / \mathrm{h}$ is assumed. These ranges are in line with the findings in [18]. Finally, two different brick types, with distinct different water absorption behaviour, are included as well for the walls with a brick veneer. The basic characteristics of the used brick types can be found in Table 3 and Figure 3. 
Postprint: Tijskens A., Roels S., Janssen H., 2019. Hygrothermal assessment of timber frame walls using a convolutional neural network. Building and Environment 193, 107652

https://doi.org/10.1016/j.buildenv.2021.107652

Table 2: Probabilistic input parameters and distributions

\begin{tabular}{ll}
\hline Parameter & Value \\
\hline Exterior climate & $\mathrm{D}(54$ European locations) \\
Exterior climate start year & $\mathrm{D}(2020 ; 2047)$ \\
Wall orientation [degrees from North] & $\mathrm{U}(0 ; 360)$ \\
Solar absorption [-] & $\mathrm{U}(0.4 ; 0.8)$ \\
Ext. heat transfer coefficient slope $k_{S}[\mathrm{~J} / \mathrm{m} 3 \mathrm{~K}]$ & $\mathrm{U}(1 ; 8)$ \\
WDR exposure factor $[-]$ & $\mathrm{U}(0 ; 2)$ \\
Cavity air change rate $[1 / \mathrm{h}]$ & Brick veneer $\mathrm{U}(2 ; 20)$ \\
Brick material & Wooden sidings $\mathrm{U}(50 ; 500)$ \\
Interior humidity load $[11]$ & $\mathrm{D}$ (Brick $1 ;$ Brick 2$)$ \\
\hline
\end{tabular}

$\mathrm{U}(\mathrm{a}, \mathrm{b})$ : uniform distribution between $\mathrm{a}$ and $\mathrm{b}$

$D(a, b)$ : discrete distribution between $a$ and $b$

Table 3: Brick type characteristics [10]

\begin{tabular}{lll}
\hline Parameter & Brick 1 & Brick 2 \\
\hline Dry thermal conductivity $\left[\mathrm{W} / \mathrm{m}^{2} \mathrm{~K}\right]$ & 0.87 & 0.52 \\
Dry vapour resistance factor $[-]$ & 139.52 & 13.25 \\
Capillary absorption coefficient $\left[\mathrm{kg} / \mathrm{m}^{2} \mathrm{~s} 0.5\right]$ & 0.046 & 0.357 \\
Capillary moisture content $\left[\mathrm{m}^{3} / \mathrm{m}^{3}\right]$ & 0.128 & 0.266 \\
Saturation moisture content $\left[\mathrm{m}^{3} / \mathrm{m}^{3}\right]$ & 0.240 & 0.367 \\
\hline
\end{tabular}

The remaining parameters are all variables either with small variations or of less importance for the current study of a 1D wall. Therefore, these boundary conditions are assumed deterministically. Table 4 gives an overview of the deterministic boundary conditions. The initial relative humidity in the timber frame construction is assumed $50 \%$, and $80 \%$ in the brick and timber cladding. The initial temperature is taken as $20^{\circ} \mathrm{C}$ throughout the entire construction. The hygrothermal simulation environment Delphin $6.0[10]$ is used to calculate the hygrothermal response. For reliable evaluation, a simulation period of four years and an hourly output frequency are adopted.

Table 4: Deterministic input parameters

\begin{tabular}{ll}
\hline Parameter & Value \\
\hline $\begin{array}{l}\text { Exterior surface } \\
\quad \text { Long wave emissivity }\end{array}$ & 0.9 \\
& \\
Interior surface & 8 \\
$\quad$ Total heat transfer coefficient $\mathrm{h}\left[\mathrm{W} / \mathrm{m}^{2} \mathrm{~K}\right]$ & $3 \times 10-8$ \\
$\quad$ Moisture transfer coefficient $\beta[\mathrm{s} / \mathrm{m}]$ & \\
& \\
Initial conditions & 20 \\
$\quad$ Initial temperature $\left[{ }^{\circ} \mathrm{C}\right]$ & 50 \\
$\quad$ Initial relative humidity $[\%]$ &
\end{tabular}


Postprint: Tijskens A., Roels S., Janssen H., 2019. Hygrothermal assessment of timber frame walls using a convolutional neural network. Building and Environment 193, 107652

https://doi.org/10.1016/j.buildenv.2021.107652

To evaluate whether a specific combination of wind and vapour barrier is adequate in a given climate, one typically analyses the relative humidity and the incidence of interstitial condensation at the interfaces between insulation and wind or vapour barrier [19]-[21] (Figure 1). A high relative humidity can entail a mould growth risk, which in this study will be assessed using the VTT mould growth model, which calculates the Mould Index based on the fluctuation of the temperature and relative humidity [22]. The Mould Index is a value between 0 and 6, going from no growth to heavy and tight mould growth. In the updated VTT model, the expected material sensitivity to mould growth is implemented as well. The materials investigated here can be categorized in the class 'sensitive'. A maximum Mould Index of 3 over the simulated period is adopted as limit state, as this equals visual mould growth and less than $10 \%$ coverage [20], [23]. To avoid interstitial condensation, the relative humidity at the interfaces should remain below $\pm 100 \%$. Additionally, a simplified criterion for the condensation rate is adopted from [19], [20], where a threshold value of $0.1 \mathrm{~kg} / \mathrm{m}^{2}$ is considered to avoid run-off. In this study, the maximum Mould Index and condensation amount over the simulated period, at the insulation - wind barrier and insulation - vapour barrier interfaces, are used as damage risk indicators.

\section{Convolutional neural network}

To reduce simulation time, this study proposes the use of a metamodel, a mathematical model that replaces the original hygrothermal model. The authors showed previously that a convolutional neural network is able to accurately capture the non-linear hygrothermal response of a massive masonry wall [6]-[8] and thus is likely to be suitable for timber frame wall simulations as well. The neural network is trained on a limited dataset, obtained with the original hygrothermal model. Afterwards, the network can be used to predict new data, based on the patterns it learned, in a much faster way than the original hygrothermal model. In the next sections, the network architecture used in [6] is adapted for the current simulation set-up, after which the network's hyper-parameters are optimised. Finally, 
Postprint: Tijskens A., Roels S., Janssen H., 2019. Hygrothermal assessment of timber frame walls using a convolutional neural network. Building and Environment 193, 107652

https://doi.org/10.1016/j.buildenv.2021.107652

the network's performance is evaluated, for both prediction accuracy and training and prediction time.

\subsection{Network architecture}

The convolutional neural network (CNN) architecture used in this study is shown in Figure 5 and is similar to the one in [6], where the authors showed that this network can accurately predict the hygrothermal performance of a massive masonry wall. The network makes use of dilated causal convolutions: the causality ensures that the network's output at a certain time step only depends on input from previous time steps, and the dilations allow the network to have a larger receptive field (i.e. the number of previous input time steps the network can access at the final layer) with fewer layers. This principle is shown in Figure 4: for a filter width of 2 and an exponentially increasing dilatation, a receptive field of 16 time steps can be obtained using only 4 layers. In contrast to the network in [6], where the filter size is kept constant over all layers, the filter size in this study is increased by its original value for each additional layer, e.g. the filter size of consecutive layers would be $3,6,9,12 \ldots$ The dilation factor is increased by multiplying with the filter size of the previous layer, e.g. the dilation factor of consecutive layers would be $1,3,18,162, \ldots$ This allows the receptive field to grow exponentially with network depth. A small-scale experiment showed that this modification, inspired on common practice in image processing, does not affect the network's prediction accuracy. It does significantly reduce the training time, however, since much fewer layers are required to obtain the same receptive field, leading to a smaller and thus faster network. The dilated causal convolutions are incorporated in residual blocks, as shown in Figure 5. Such a residual block consists of a dilated causal convolution, followed by a gated activation unit, a skip connection and a residual connection. The gated activation unit controls the flow of information through the residual block, while the skip connection allows lower level signals to pass unfiltered to the final layers of the network, which in turn allows the network to identify different aspects of the time series (i.e. strong autoregressive components, sophisticated trend and seasonality components). The residual connection, to end, 
Postprint: Tijskens A., Roels S., Janssen H., 2019. Hygrothermal assessment of timber frame walls using a convolutional neural network. Building and Environment 193, 107652

https://doi.org/10.1016/j.buildenv.2021.107652

facilitates training deeper networks by bypassing the gated activation unit [24]. By layering these residual blocks, the receptive field can increase exponentially, as the filter size and dilation rate increase. A combination of layered residual blocks is called a stack, which can also be repeated. For each new stack, the filter size starts again from the initial filter size and the dilatation rate starts from one. Using multiple stacks allows for a more complex network, without reaching a receptive field that is much larger than the actual sequence length.

The main input to the network is a tensor containing the interior and exterior climate time series. In Figure 5, this network input is denoted 'sequence input (climate time series)'. Additionally, to enable the network to produce output patterns for a specific context, the input to the gated activation unit is conditioned on a tensor that contains contextual time-invariant information. In Figure 5 , this input tensor is denoted 'scalar input'. This conditioning tensor contains the scalar input variables such as the wall orientation, wall thickness or single-valued material characteristics, broadcasted over the time dimension. In this study, the materials' moisture transport and storage characteristics play an additional role in the hygrothermal performance of timber frame walls. Therefore, in contrast to previous studies [6], [7], an additional input tensor is added to the conditioning tensor, which contains the materials moisture properties and is denoted as 'sequence input (material data series)' in Figure 5. In this study, this tensor contains the materials' moisture retention curve (Figure 2) and relativehumidity-dependent vapour resistance (Figure 3), as these have the largest influence on the hygrothermal response. Both functions are represented by 11 points, corresponding to either the capillary pressure from $1^{0}$ to $1^{10}$ or the relative humidity from 0 to 100 . Since this is a sequence input tensor, it is first passed through a convolution with a filter size equal to the length of the sequences (in this case, 11) to allow concatenating with the scalar input tensor. 
Postprint: Tijskens A., Roels S., Janssen H., 2019. Hygrothermal assessment of timber frame walls using a convolutional neural network. Building and Environment 193, 107652

https://doi.org/10.1016/j.buildenv.2021.107652

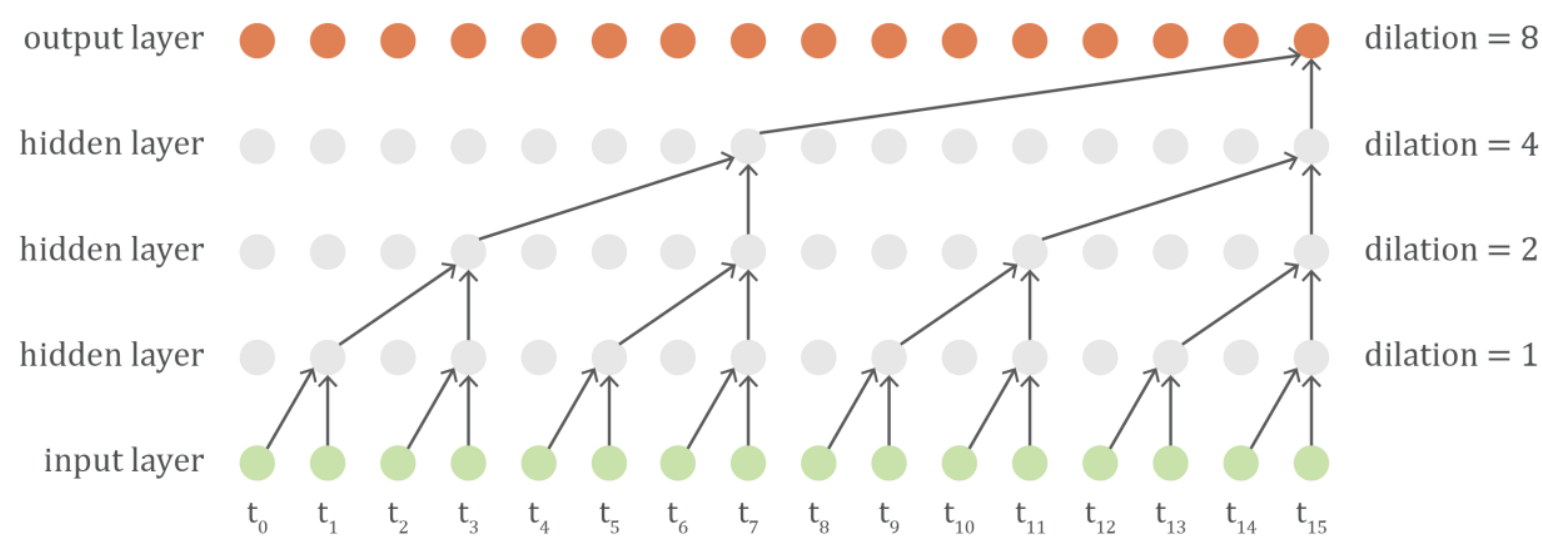

Figure 4: The causal dilated convolutions allow an output time step to receive information from a larger range of input time steps (i.e. the receptive field) with increasing number of hidden layers. In the presented scheme, a filter width of two, four layers and one stack result in a receptive field of sixteen input steps.

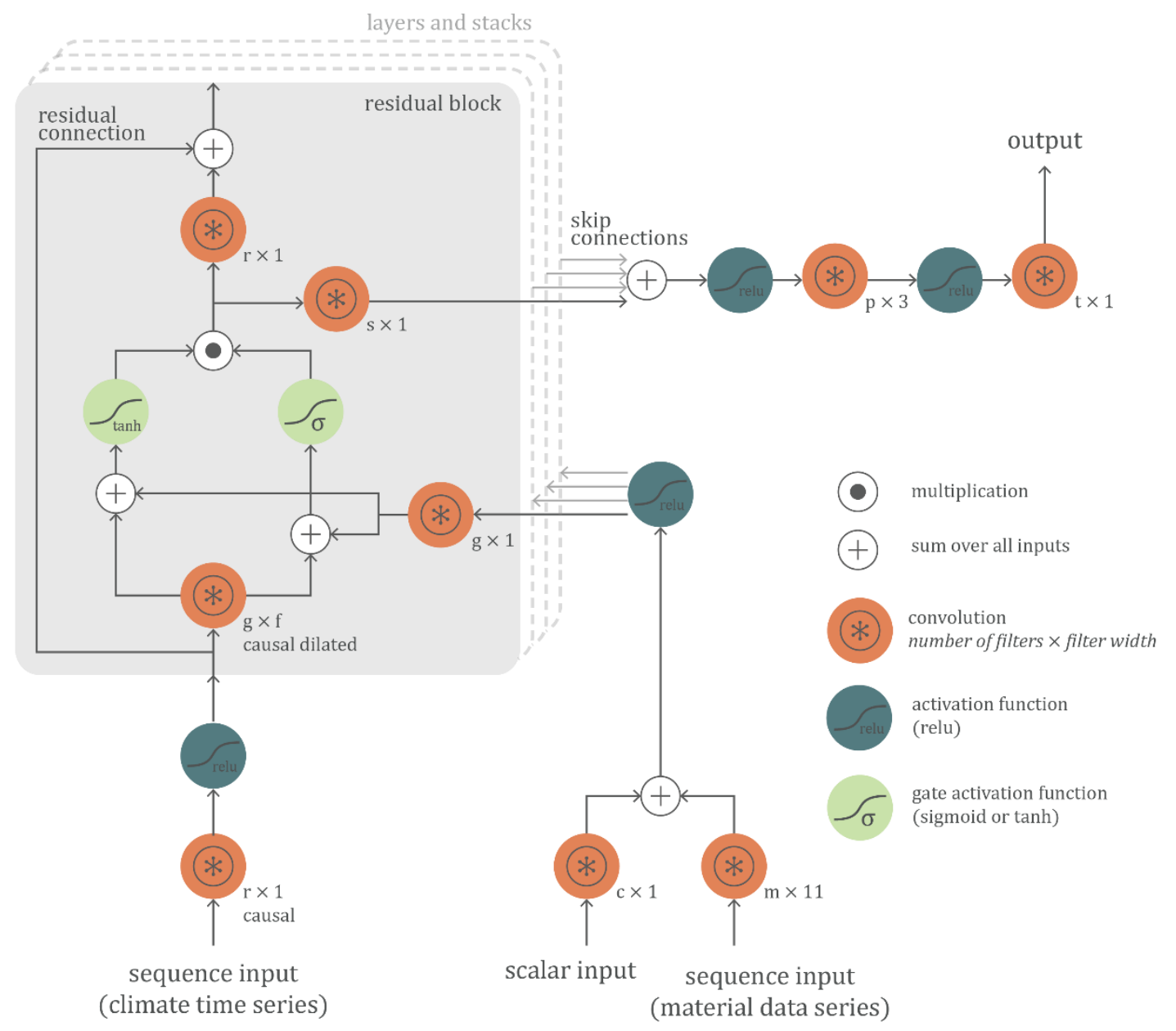

Figure 5: The used CNN architecture with residual blocks, skip connections and global conditioning. 
Postprint: Tijskens A., Roels S., Janssen H., 2019. Hygrothermal assessment of timber frame walls using a convolutional neural network. Building and Environment 193, 107652

https://doi.org/10.1016/j.buildenv.2021.107652

To train the network, it is presented with input-output data pairs; the input data is sampled from the input distributions given in section 2 , the output data is obtained by simulating these samples with the original hygrothermal model. Before presenting the input and output data to the neural network, all data are standardised (zero mean, unit variance). Standardising the input data ensures that all features are on the same scale, which allows weighting them equally in their representation. Standardising the output data ensures that errors are penalised equally for all targets.

\subsection{Hyper-parameter optimisation}

\section{Methodology}

Before the network can be trained, the hyper-parameters need to be determined. These are model configuration parameters that are external to the model and whose value cannot be estimated from the training data, such as the number of layers, stacks, filters, the size of the initial filter width, as well as the loss function, learning rate, etc. The loss function, learning algorithm, learning rate, number of training epochs and batch size concern the training process. As previously in [6], the root-meansquared-error (equation (2)) is chosen as loss function and the Adam algorithm [25] is used as learning algorithm.

$$
R M S E=\sqrt{\frac{1}{N} \sum(y-\hat{y})^{2}}
$$

The learning rate defines how much the neurons' weights can change after each step of the learning process. Too large weight updates might cause the network's performance to oscillate over training epochs, whereas too small weight updates might cause the training algorithm to become stuck in local optima. Therefore, the learning rate must carefully be fine-tuned. The batch size defines the number of samples the network is trained on at the same time. After each batch, the network's weights are updated; a smaller batch size results in more weight updates. Previous experiments showed that a batch size of 4 is most optimal [6]. When all batches are passed through the network once, one training epoch is completed. The number of training epochs is the number of times the network is exposed to the entire training dataset. The more epochs, and thus exposure to the training data, the better the network can learn a correct mapping. However, too much exposure can lead to overfitting: the network's error on the training data is small but when new data is presented to the network, the error 
Postprint: Tijskens A., Roels S., Janssen H., 2019. Hygrothermal assessment of timber frame walls using a convolutional neural network. Building and Environment 193, 107652

https://doi.org/10.1016/j.buildenv.2021.107652

is large. Overfitting can be prevented by using a mechanism called 'early stopping': training is stopped once the error on the validation dataset no longer decreases. As in [6], during the hyper-parameter optimisation, a maximum of 50 epochs is imposed, and training is stopped earlier if the RMSE on the validation set decreases less than 0.001 over 5 epochs. Thus, the only hyper-parameter related to the training process that needs fine-tuning is the learning rate. Additionally, there are hyper-parameters related to the network architecture itself (Figure 5). Table 5 gives an overview of all hyper-parameters and their optimisation search range.

Table 5: The search range of the hyper-parameters

\begin{tabular}{ll}
\hline Hyper-parameter & Range \\
\hline Number of filters $c$ & {$\left[2^{5} ; 2^{9}\right]$} \\
Number of filters $m$ & {$\left[2^{5} ; 2^{9}\right]$} \\
Number of filters $g$ & {$\left[2^{5} ; 2^{9}\right]$} \\
Number of filters $s$ & {$\left[2^{5} ; 2^{9}\right]$} \\
Number of filters $r$ & {$\left[2^{5} ; 2^{9}\right]$} \\
Number of filters $p$ & {$\left[2^{5} ; 2^{9}\right]$} \\
Initial filter width $f$ & {$[2 ; 10]$} \\
Number of layers & {$[2 ; 6]$} \\
Number of stacks & {$[1 ; 4]$} \\
Learning rate & {$[0.0001 ; 0.01]$} \\
\hline
\end{tabular}

The same Grey Wolf Optimiser (GWO) [26] as in [6] is used, as it was proven an efficient alternative to a full-factorial evaluation of all possible hyper-parameter combinations. GWO is a population-based meta-heuristic optimisation algorithm, based on the leadership hierarchy and hunting mechanism of grey wolves in nature. In search of the optimal solution, the three fittest solutions guide the direction of the search agents, by updating their positions according to the fittest positions. The fitness of a solutions is defined by the RMSE on the validation dataset. Again, 10 search agents are deployed to explore and exploit the search space over 100 iterations. This is repeated for five independent runs, as different runs might end up with different optimal solutions. To reduce the training time during the optimisation process, the training dataset contains only 672 samples and the validation dataset 288 samples. 
Postprint: Tijskens A., Roels S., Janssen H., 2019. Hygrothermal assessment of timber frame walls using a convolutional neural network. Building and Environment 193, 107652

https://doi.org/10.1016/j.buildenv.2021.107652

All networks in this study are trained and evaluated on a machine with a dual NVIDIA RTX2080 GPU with 2x8GB memory.

\section{Results and discussion}

The hyper-parameter optimisation shows very similar results as found in [6], where a similar network, trained on a massive masonry wall, is optimised. Figure 6 shows the RMSE on the validation dataset of all GWO candidate solutions, in function of the receptive field and the initial filter width of the causal dilated convolution. This figure clearly shows that a receptive field of at least 11 months (8000 hours) is required to obtain low RMSE. The red line indicates the lower edge of the candidate solutions found in [6], when optimising the network for a massive masonry wall. It shows that the receptive field's threshold is lower when predicting the hygrothermal response of a timber frame walls, compared to predicting the hygrothermal response of massive masonry walls. This can be explained by the fact that a timber frame wall simply responds faster to changing boundary conditions than a massive masonry wall does, and that events from a distant past (e.g. a heavy rain shower) have lower impact on the current hygrothermal state. Furthermore, note that a larger receptive field generally results in a larger number of network parameters, and thus in an increased training time. Hence, it is not beneficial to use a receptive field that is (much) larger than the time series length.

Similar to [6], using multiple stacks rather than one, slightly lowers the RMSE on the validation set but increases the training time (results not shown here), while the number of layers has the same influence on the training time (results not shown here), but not on the prediction performance provided that the receptive field is large enough. Furthermore, no significant relations between the number of filters and the prediction performance were found, though again, the number of filters does influence the number of parameters, which in turn affects the training time.

Finally, Figure 7 shows that the optimal learning rate for this network architecture lies between 0.001 and 0.0025 . Unlike in [6], no relation between learning rate and filter width was found, which can be 
Postprint: Tijskens A., Roels S., Janssen H., 2019. Hygrothermal assessment of timber frame walls using a convolutional neural network. Building and Environment 193, 107652

https://doi.org/10.1016/j.buildenv.2021.107652

explained by the increasing filter width used in the current network architecture, while the filter width was kept constant in [6].

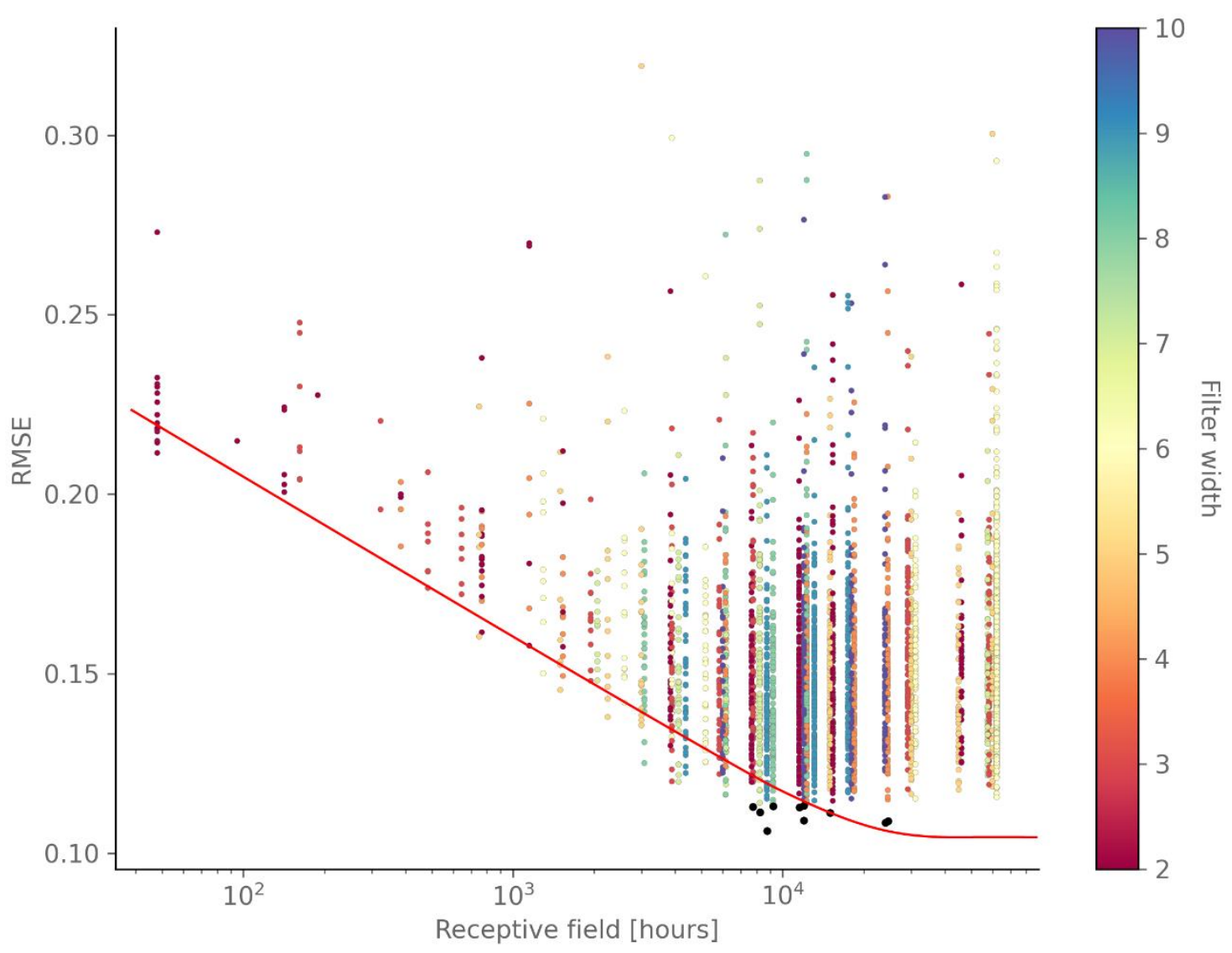

Figure 6: This scatterplot of all GWO candidate solutions shows that a receptive field of at least 11 months ( 8000 hours) is required to obtain a low RMSE on the validation set, and that a low RMSE can be obtained for all filters widths, once above this receptive field threshold. The 10 best solutions are indicated in black. The red line indicates the lower edge of the results found in [6] when optimising a CNN for hygrothermal simulations on a massive masonry wall. 
Postprint: Tijskens A., Roels S., Janssen H., 2019. Hygrothermal assessment of timber frame walls using a convolutional neural network. Building and Environment 193, 107652

https://doi.org/10.1016/j.buildenv.2021.107652

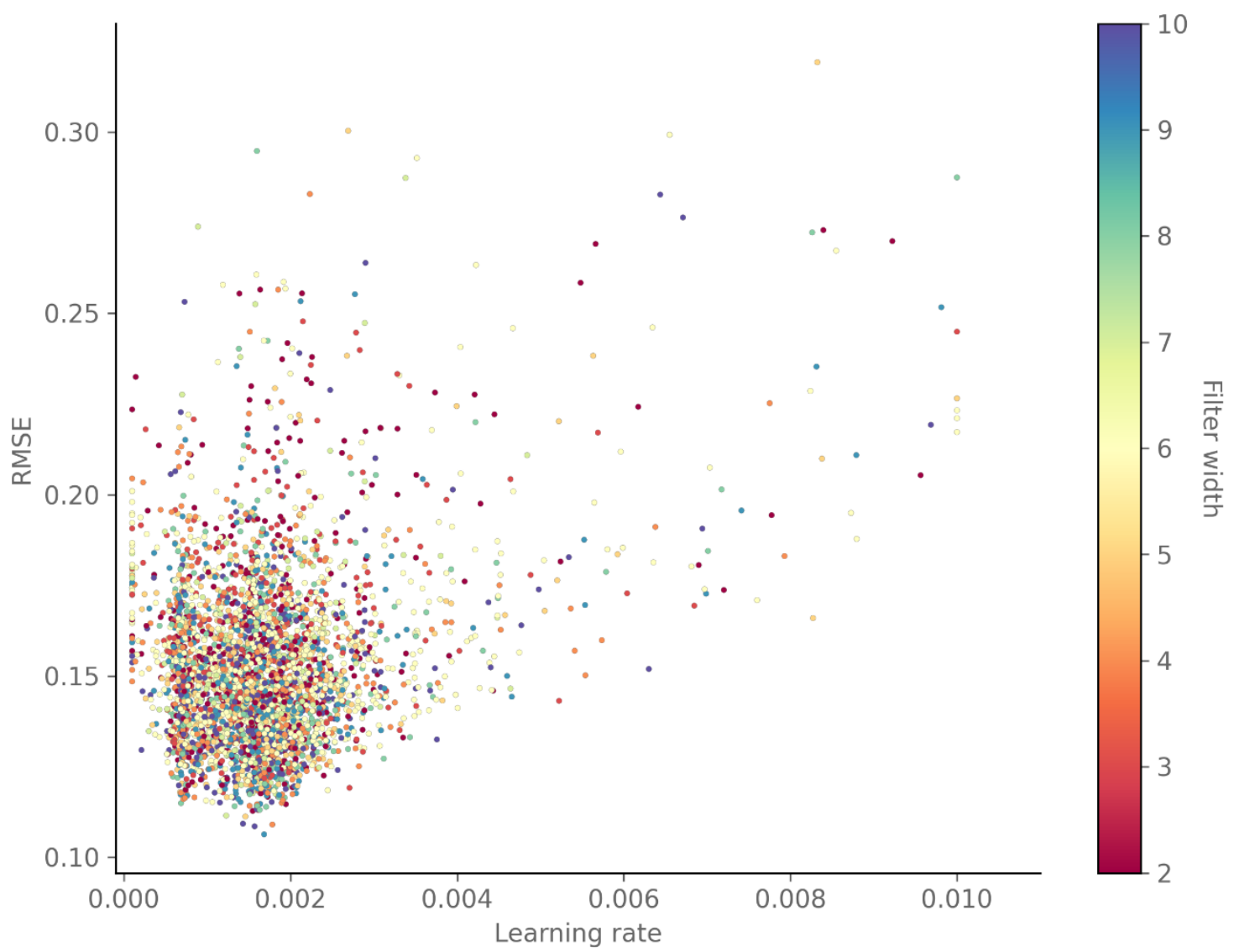

Figure 7: This scatterplot of all GWO candidate solutions shows that the optimal learning rate lies between 0.001 and 0.003 and does not depend on the filter width.

Overall, the same conclusions as drawn in [6] are valid here as well. For the current network architecture, they can be summarised as follows:

- The receptive field should be large enough (>11 months) but not (much) larger than the time series length.

- Use fewer layers and stacks to reduce training time. A larger (initial) filter width allows using fewer layers and stacks to obtain the same receptive field.

- Use a low number of filters, to reduce training time, but not too low to allow complexity (64256).

- Use a learning rate between 0.001 and 0.0025 .

Hence, when following these 'rules of thumb', it might not be necessary to perform such an extensive hyper-parameter optimisation as performed in this study and in [6]. Between [6], where the network is optimised for a massive masonry wall, and this study, where the network is optimised for a lightweight timber frame wall, most common types of wall constructions are covered. Hence, the above- 
Postprint: Tijskens A., Roels S., Janssen H., 2019. Hygrothermal assessment of timber frame walls using a convolutional neural network. Building and Environment 193, 107652

https://doi.org/10.1016/j.buildenv.2021.107652

mentioned guidelines can simply be applied to most wall constructions without having to perform a hyper-parameters optimisation again. Depending on the case study, it might be beneficial to explore the right size of receptive field, as it has a significant impact on the prediction performance and on the training time.

\subsection{Performance evaluation}

\section{Methodology}

During the hyper-parameter optimisation, the networks were trained for maximum 50 epochs only and on a limited dataset and as a result, it was not possible to obtain maximum prediction performance. Therefore, the ten best solutions (with the lowest validation RMSE) of all runs done in relation to the hyper-parameter optimisation are trained again using a larger dataset, containing 5760 samples in total. To ensure the final network generalises well, $K$-fold cross-validation is applied. Figure 8 shows a flowchart of this technique, which involves splitting the dataset in $K$ non-overlapping subsets (folds) and performing $K$ rounds of training, validating and testing. Each round, two subsets are used as validation and testing set respectively, with the remaining $K-2$ subsets acting as training set. Only the training and validation set are used to train the network: the training set is used to update the networks weights, while the validation set is used to evaluate when training needs to stop (early stopping). A maximum of 200 epochs is set, with early stopping if the RMSE on the validation set decreases less than 0.001 over 25 epochs. The testing set is used to evaluate the network's performance on unseen data. This approach thus allows estimating the generalisation error, which is a measure of how accurately the network is able to predict for previously unseen data. The networks' performance is evaluated on the test set using the root mean square error (RMSE) and the mean absolute error (MAE) as performance indicators, which are quantified as follows:

$$
R M S E=\sqrt{\frac{1}{N} \sum(y-\hat{y})^{2}} \quad M A E=\frac{1}{N} \sum|y-\hat{y}|
$$

where $y$ is the true output, $\hat{y}$ is the predicted output and $N$ is the total number of data points. These indicators are calculated on the standardised data (zero mean, unit variance), as this ensures that 
Postprint: Tijskens A., Roels S., Janssen H., 2019. Hygrothermal assessment of timber frame walls using a convolutional neural network. Building and Environment 193, 107652

https://doi.org/10.1016/j.buildenv.2021.107652

errors are penalised equally for all targets. Finally, the generalisation error can be estimated by averaging the performance indicators (RMSE and MAE) of all $K$ folds.

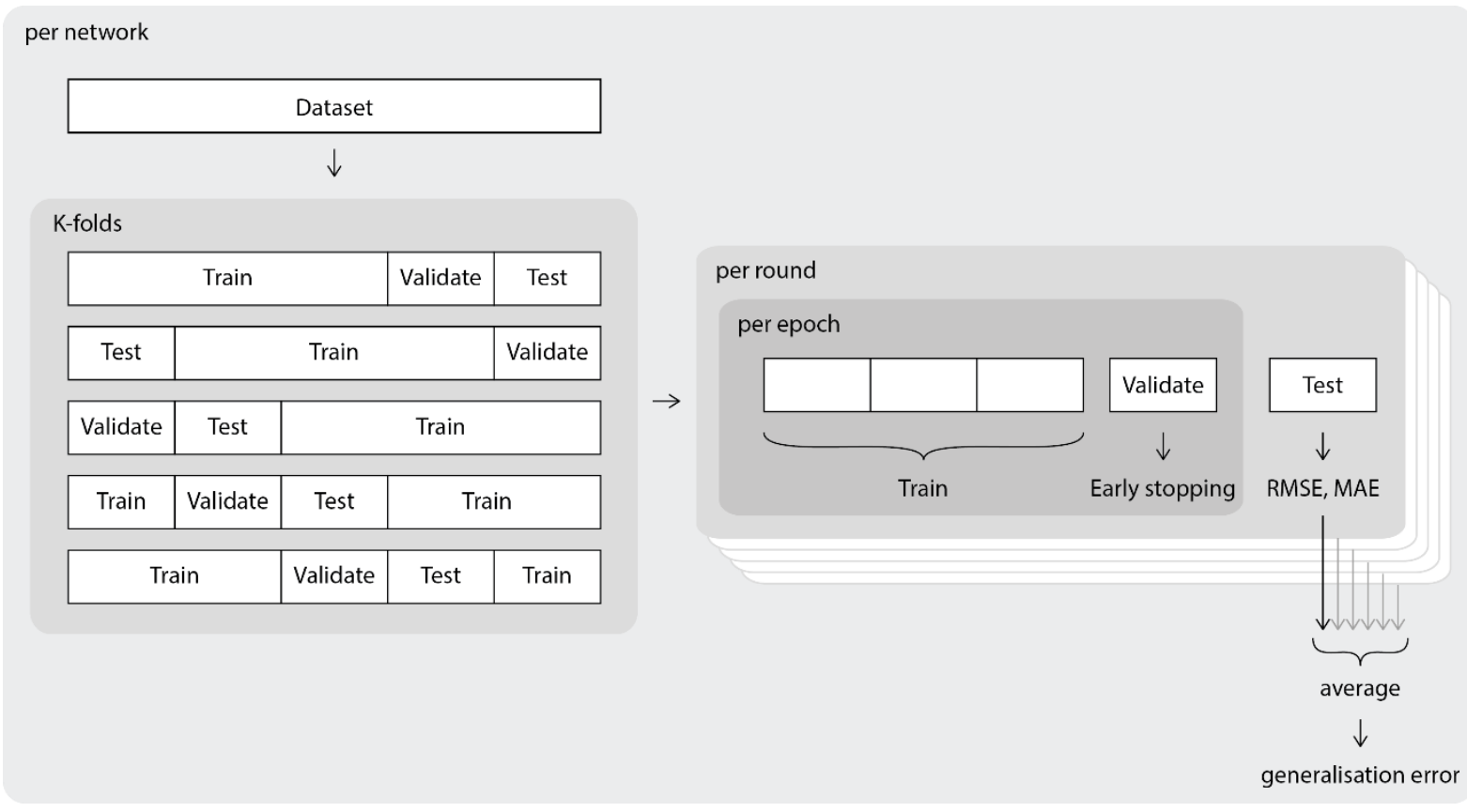

Figure 8: Flowchart of $K$-fold cross-validation.

In this study, a five-fold cross-validation is applied, resulting in a training set of 3456 samples and a validation and testing set of each 1152 samples. Besides the RMSE and MAE, the networks' training time is evaluated as well. After cross-validation, the best performing network, defined as the one with the lowest average RMSE (i.e. the lowest generalisation error), is selected. This final network will be used in section 4 to evaluate the timber frame wall's hygrothermal performance.

\section{Results and discussion}

The ten best solutions for the hyper-parameters, as determined by the Grey Wolf Optimiser algorithm, are shown in Table 6, while Figure 9 shows these networks' cross-validation performance indicators. Clearly, the generalisation errors of all networks are quite close to each other. These results confirm the overall findings described above, i.e. there is no single hyper-parameter combination that significantly outperforms the others. As long as the training dataset contains enough samples, the receptive field is large enough (> 11 months) and a learning rate between 0.001 and 0.0025 is chosen, the other hyper-parameters appear to have only a minor influence on the prediction performance. 
Postprint: Tijskens A., Roels S., Janssen H., 2019. Hygrothermal assessment of timber frame walls using a convolutional neural network. Building and Environment 193, 107652

https://doi.org/10.1016/j.buildenv.2021.107652

Table 6: The ten best performing solutions of the Grey Wolf Optimiser algorithm.

\begin{tabular}{lrrrrrrrrrr}
\hline & $\begin{array}{c}\text { Conditional } \\
\text { filters }\end{array}$ & $\begin{array}{c}\text { Material } \\
\text { filters }\end{array}$ & $\begin{array}{c}\text { Gate } \\
\text { filters }\end{array}$ & $\begin{array}{c}\text { Skip } \\
\text { filters }\end{array}$ & $\begin{array}{c}\text { Residual } \\
\text { filters }\end{array}$ & $\begin{array}{c}\text { Penultimate } \\
\text { filters }\end{array}$ & $\begin{array}{c}\text { Initial } \\
\text { filter } \\
\text { width }\end{array}$ & Layers & Stacks & $\begin{array}{c}\text { Learning } \\
\text { rate }\end{array}$ \\
\hline 1 & 256 & 128 & 512 & 512 & 256 & 32 & 9 & 2 & 2 & 0,00168 \\
2 & 128 & 64 & 512 & 128 & 128 & 512 & 10 & 2 & 4 & 0,00157 \\
3 & 256 & 512 & 128 & 256 & 256 & 64 & 4 & 3 & 4 & 0,00178 \\
4 & 64 & 512 & 512 & 256 & 64 & 64 & 10 & 2 & 2 & 0,00143 \\
5 & 32 & 256 & 512 & 128 & 512 & 64 & 5 & 3 & 1 & 0,00146 \\
6 & 64 & 512 & 256 & 256 & 32 & 32 & 7 & 2 & 4 & 0,00123 \\
7 & 64 & 512 & 256 & 256 & 64 & 64 & 2 & 4 & 3 & 0,00155 \\
8 & 128 & 512 & 128 & 256 & 32 & 64 & 3 & 3 & 4 & 0,00161 \\
9 & 512 & 512 & 128 & 128 & 512 & 32 & 8 & 2 & 3 & 0,00163 \\
10 & 64 & 512 & 256 & 256 & 64 & 64 & 10 & 2 & 2 & 0,00160 \\
\hline
\end{tabular}

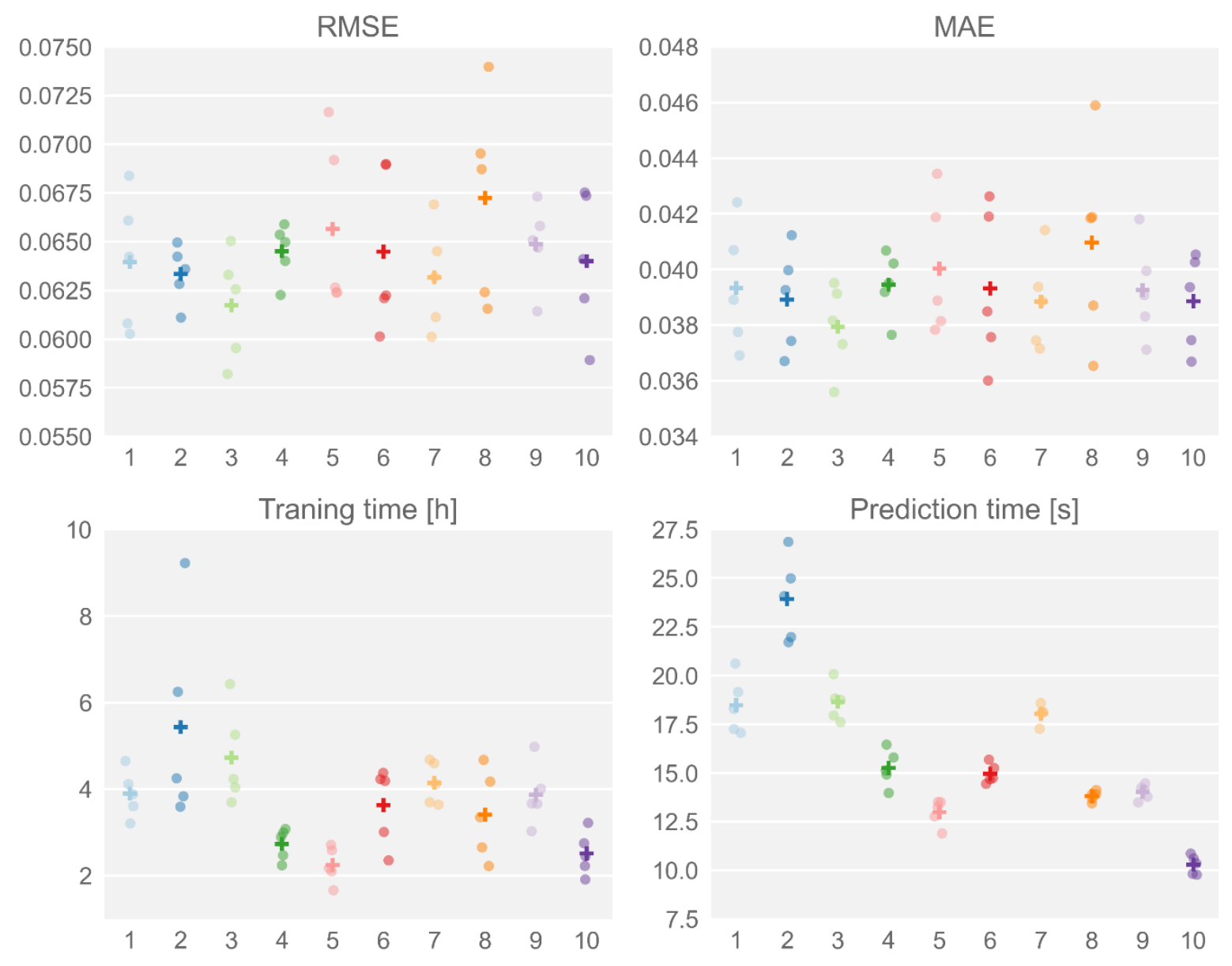

Figure 9: The performance indicators, training time and prediction time for the ten best performing hyper-parameter combinations, after being trained fully. For each combination, the dots represent one cross-validation fold, whereas the cross indicates the average over all five folds.

Figure 9 shows that all networks have a very similar generalisation error, which indicates the GWO algorithm successfully put the best performing networks forward. Regarding training time, there is a 
Postprint: Tijskens A., Roels S., Janssen H., 2019. Hygrothermal assessment of timber frame walls using a convolutional neural network. Building and Environment 193, 107652

https://doi.org/10.1016/j.buildenv.2021.107652

quite large variation between the different networks. As described in the previous section, this is caused by a differing number of stacks, levels and filters. The prediction time is the time required for the network to predict a certain number of samples. The prediction time shown in Figure 9 is for the 1152 samples of the test fold. A similar trend as for the training time can be observed, i.e. networks with fewer trainable parameters can predict faster. However, the time gained by selecting a network that predicts faster is very limited as the difference is only a few seconds. Hence, in most applications, it is more important to focus on a network with high prediction accuracy and perhaps a low training time, than on a network with low prediction time. The third network has the lowest generalisation error (average RMSE) and is thus selected as best performing. Note that networks 4 and 10 perform almost equally good but have a much lower training time than network 3 . This again proves that smaller networks can have good performance and thus might be preferable when training time is an issue and no hyper-parameter optimisation is performed.

Once the network is trained, it can be used to predict new data. To predict 1152 test set samples, the network needed about 24 seconds on a machine with a dual NVIDIA RTX 2080 8GB GPU. The same simulations using the original hygrothermal model took about 3.5 hours on a machine with a dual Intel Xeon E5-2665 CPU (16 cores with 32 threads). This means that, for the current machine setup, the neural network is over 500 times faster. Of course, one should take into account the time required to create the training dataset and to train the neural network. Yet, when one wishes to evaluate a large number of wall types and/or a large number of samples with a lot of variability, it is clear that the neural network will be much more time efficient. Note also that the current GPU setup is not the most efficient one available. GPU's with a larger memory would allow a faster training and predicting, thus even increasing the time savings. This becomes especially interesting when training on even larger datasets (e.g. more wall types or more probabilistic input parameters with more variability).

In Figure 10, the network's hygrothermal predictions (orange) are shown for one sample that the network was not trained on, and are compared to the true hygrothermal values (blue). The 
Postprint: Tijskens A., Roels S., Janssen H., 2019. Hygrothermal assessment of timber frame walls using a convolutional neural network. Building and Environment 193, 107652

https://doi.org/10.1016/j.buildenv.2021.107652

performance indicators shown above each panel are calculated on the standardised output for each target separately, as this indicates the difference in accuracy between targets. It is clear that the chosen network is able to predict all hygrothermal outputs quite accurately. As described at the end of section 2 , these hygrothermal predictions can be used to evaluate damage risks. For this study, the damage risk indicators are the maximum Mould Index and the maximum condensation rate, at the interfaces between insulation and wind or vapour barrier. Figure 11 shows the damage risk indicators for all test set samples using the networks' output ( $y$-axis), compared to the true values ( $x$-axis), as calculated using the outputs of the hygrothermal model. This shows that the damage risk prediction, based on the networks' hygrothermal predictions, is quite accurate for the vast majority of the test set samples. It appears that predictions for the condensation rate at the interface between insulation and wind barrier are less accurate as the condensation rate is higher $\left(>0.5 \mathrm{~kg} / \mathrm{m}^{2}\right)$. However, since the limit is set to $0.1 \mathrm{~kg} / \mathrm{m}^{2}$ to avoid moisture damage, this will likely not influence the choice of most optimal wall composition. Furthermore, the network tends to underestimate the condensation rate at the interface between insulation and vapour barrier in the range $>0.1 \mathrm{~kg} / \mathrm{m}^{2}$. Since the dataset contains very few samples were the condensation rate exceeds $0.1 \mathrm{~kg} / \mathrm{m}^{2}$ (only 15 on a total of 5760 ), the network is not able to capture this pattern accurately. If accuracy in this range is important, a solution could be to penalise errors on the condensation rate more, thus forcing the network to adapt its weights in favour of a more accurate approximation of this output. Unfortunately, this might also lead to a decrease in accuracy for other output parameters. Furthermore, based on an analysis of data obtained with the original hygrothermal model, it appears that high condensation rates at the vapour barrier is a marginal event, occurring only seldom and only in cases where also much higher condensation rates at the wind barrier occurs. Therefore, this reduced accuracy is unlikely have a significant impact on the choice of most optimal wall composition.

One could interpret the damage criteria also as a classification problem, though this is a rather strict judgement. Table 7 indicates the percentage of samples that was classified correctly, the percentage 
Postprint: Tijskens A., Roels S., Janssen H., 2019. Hygrothermal assessment of timber frame walls using a convolutional neural network. Building and Environment 193, 107652

https://doi.org/10.1016/j.buildenv.2021.107652

of false negatives (wrongfully classified below the damage limit) and the percentage of false positives (wrongfully classified above the damage limit $-\mathrm{MI}<3 ; \mathrm{CR}<0.1 \mathrm{~kg} / \mathrm{m}^{2}$ ). Clearly, the number of false negative or false positive samples is really small. This indeed proves that the inaccuracies observed above are unlikely to influence the choice of most optimal wall composition, and that one can employ the network with confidence.
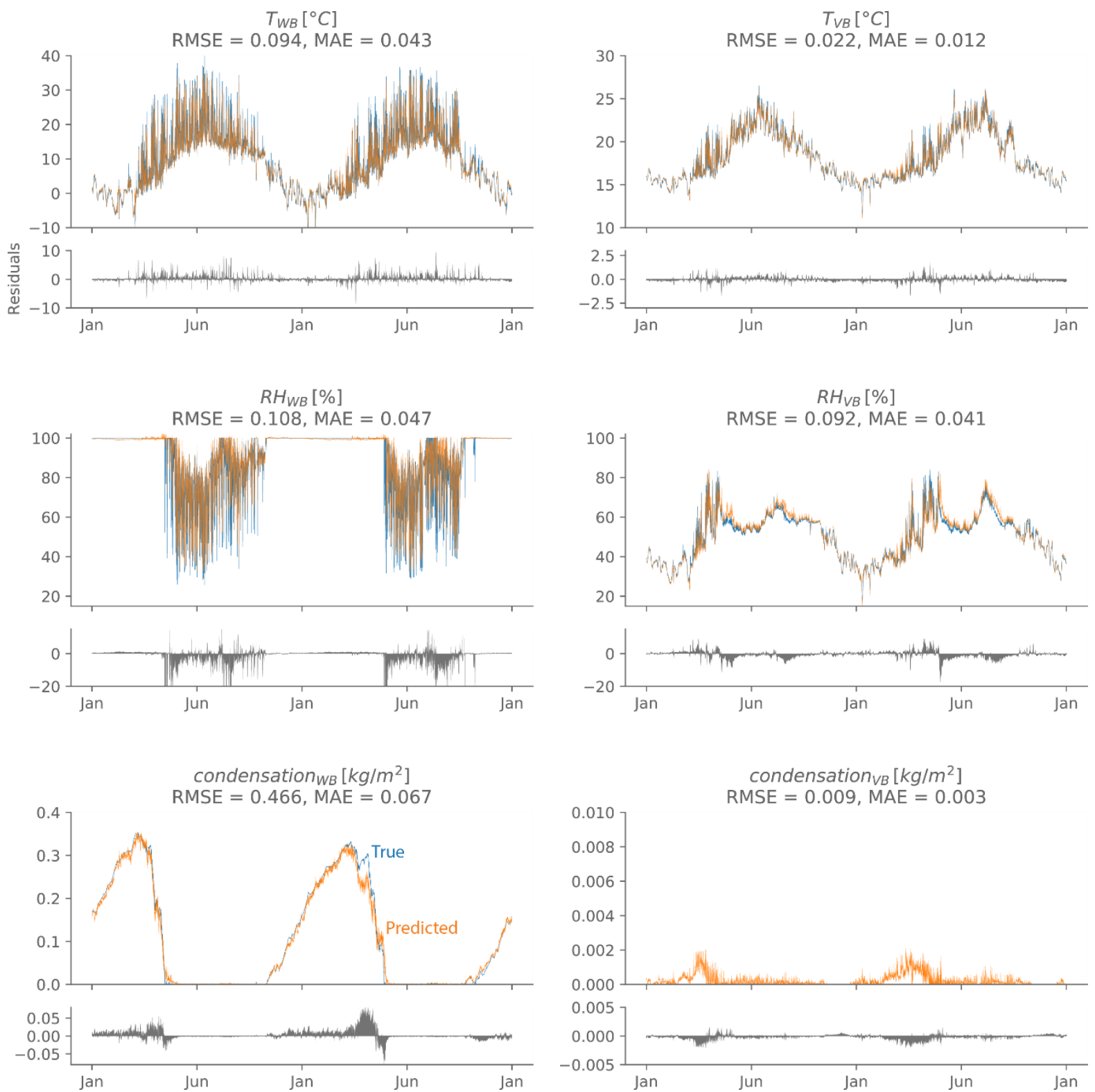

Figure 10: The network's hygrothermal predictions (orange) of one test set sample. The true value is shown in blue, the prediction errors (residuals) are indicated in grey. 
Postprint: Tijskens A., Roels S., Janssen H., 2019. Hygrothermal assessment of timber frame walls using a convolutional neural network. Building and Environment 193, 107652

https://doi.org/10.1016/j.buildenv.2021.107652
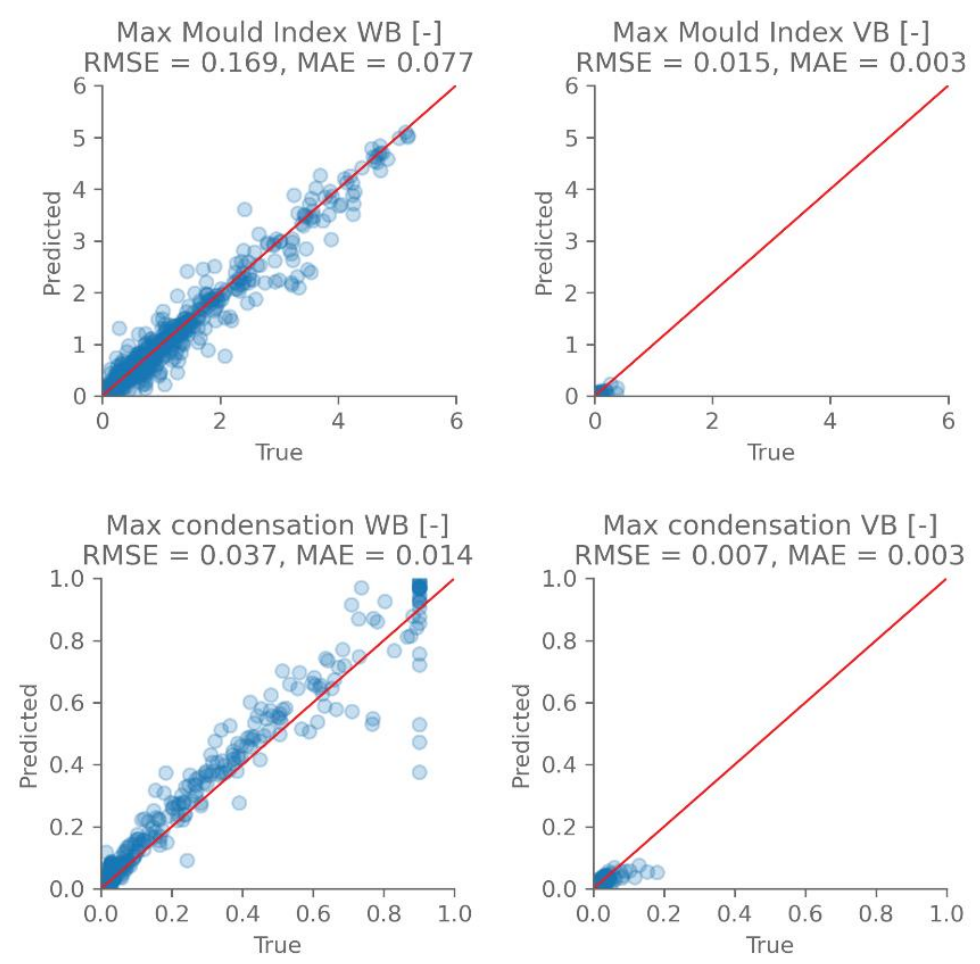

Figure 11: A comparison between the damage risk indicators for all samples, as calculated by using the neural network's predictions ( $y$-axis) and the true hygrothermal simulation output ( $x$-axis).

Table 7: The percentage of samples classified correctly, as false negative or as false positive.

\begin{tabular}{lcccc} 
& $\begin{array}{c}\text { Max Mould } \\
\text { Index WB }\end{array}$ & $\begin{array}{c}\text { Max Mould } \\
\text { Index VB }\end{array}$ & $\begin{array}{c}\text { Max condensation } \\
\text { WB }\end{array}$ & $\begin{array}{c}\text { Max condensation } \\
\text { VB }\end{array}$ \\
\hline Correct & $98.9 \%$ & $100 \%$ & $99.4 \%$ & $99.7 \%$ \\
False negative & $0.9 \%$ & $0 \%$ & $0 \%$ & $0.3 \%$ \\
False positive & $0.2 \%$ & $0 \%$ & $0.6 \%$ & $0 \%$ \\
\hline False negative: wrongfully classified below damage threshold & & \\
False positive: wrongfully classified above damage threshold & & \\
\hline
\end{tabular}

\section{Application as a decision tool}

Now the network is developed and its' accuracy has been verified, it can be put to use and predict new data. This stage is called inference. To evaluate the hygrothermal performance of the 96 different wall types described in section 2, the network is used to propagate the uncertainty and variability of the input parameters to probability distributions of the performance indicators, by simulating 128 samples per wall type. A multilayered sampling scheme [27] is used, so that each wall type is evaluated using the same 128 sampled input parameter combinations. Hence, each wall type is subjected to the same uncertainties, allowing a reliable comparison of the different wall types' hygrothermal performance. As a result, 12288 simulations were carried out in total (96 wall types $x 128$ samples). 
Postprint: Tijskens A., Roels S., Janssen H., 2019. Hygrothermal assessment of timber frame walls using a convolutional neural network. Building and Environment 193, 107652

https://doi.org/10.1016/j.buildenv.2021.107652

The network needed 4 minutes to complete these, on a machine with a dual NVIDIA RTX2080 GPU with $2 \times 8 G B$ memory. If the same amount of samples were simulated using the original hygrothermal model, this would have taken roughly 1.5 days on a machine with a dual Intel Xeon E5-2665 CPU (16 cores with 32 threads).

As described in section 2, the hygrothermal performance of the timber frame walls is evaluated by estimating the mould growth and interstitial condensation risk. To avoid damage caused by mould growth, the maximum Mould Index should not be higher than 3, which corresponds to visual mould growth and a surface coverage less than $10 \%$. To avoid run-off caused by interstitial condensation, the condensation rate should not exceed $0.1 \mathrm{~kg} / \mathrm{m}^{2}$. The results showed no risk for mould growth and runoff at the interface between insulation and vapour barrier, regardless of wall type or climate. Note that this might be an underestimation due to the lower accuracy in the range $>0.1 \mathrm{~kg} / \mathrm{m}^{2}$. As explained above, this is unlikely to influence the optimal choice of wall composition though. Hence, these results are neither shown nor expounded.

Current guidelines focus on the $s_{d^{-}}$ratio between wind and vapour barrier. For many barriers, the $s_{d^{-}}$ value varies with the relative humidity though (see Figure 3), which complicates calculating the ratio. In this study, the $s_{d}$-ratio is therefore calculated using the $s_{d}$-value at the average exterior and interior relative humidity during the heating season (September $1^{\text {st }}-$ April $31^{\text {st }}$ ), for the wind and vapour barrier respectively. Note that these average relative humidities vary as well, depending on the climate. As a result, the $s_{d}$-ratios should be calculated per climate, or, for simplification, per KöppenGeiger climate classes [13]. This paper focuses on the Belgian and Finnish climates, as currently guidelines are available only for these countries. Belgium's climate belongs to climate class Cfb (temperate oceanic climate), for which an average exterior relative humidity of $80 \%$ and average interior relative humidity of $55 \%$ were found. Finland's climate predominantly belongs to climate class Dfc (subarctic climate), for which an average exterior relative humidity of $90 \%$ and average interior relative humidity of $50 \%$ were found. The results for other climate classes will not be shown here. 
Postprint: Tijskens A., Roels S., Janssen H., 2019. Hygrothermal assessment of timber frame walls using a convolutional neural network. Building and Environment 193, 107652

https://doi.org/10.1016/j.buildenv.2021.107652

For climate class Cfb, Figure 12 and Figure 13 show the maximum Mould Index and condensation rate respectively, in function of the $s_{d}$-ratio. The Belgian Building Research Institute [2] proposes an $s_{d^{-}}$ ratio of at least 15 for walls with a brick veneer. For walls with a wooden cladding, a $s_{d}$-ratio of at least 6 was put forward. These results indicate this limit is appropriate, albeit a bit conservative, and that the distinction between cladding type is justified. Furthermore, Figure 12 shows that the Mould Index is lower when sidings are used, which is likely due to the higher ventilation rate of the air cavity between cladding and wind barrier. The impact of the cladding type is much less noticeable on the condensation risk, shown in Figure 13, where a different trend can be observed. When the wall is insulated with cellulose, run-off does not occur, regardless of the used barriers. On the other hand, if mineral wool is used, there is clearly a high condensation risk when combined with non-buffering wind barriers (i.e. foils) but not otherwise. Cellulose insulation has a high moisture buffering capacity (see Figure 2) and thus is able to buffer and redistribute all excess moisture easily. Mineral wool does not allow hygric buffering, so the wind barrier must be able to buffer the excess moisture. This excludes the use of wind barrier foils. 
Postprint: Tijskens A., Roels S., Janssen H., 2019. Hygrothermal assessment of timber frame walls using a convolutional neural network. Building and Environment 193, 107652

https://doi.org/10.1016/j.buildenv.2021.107652
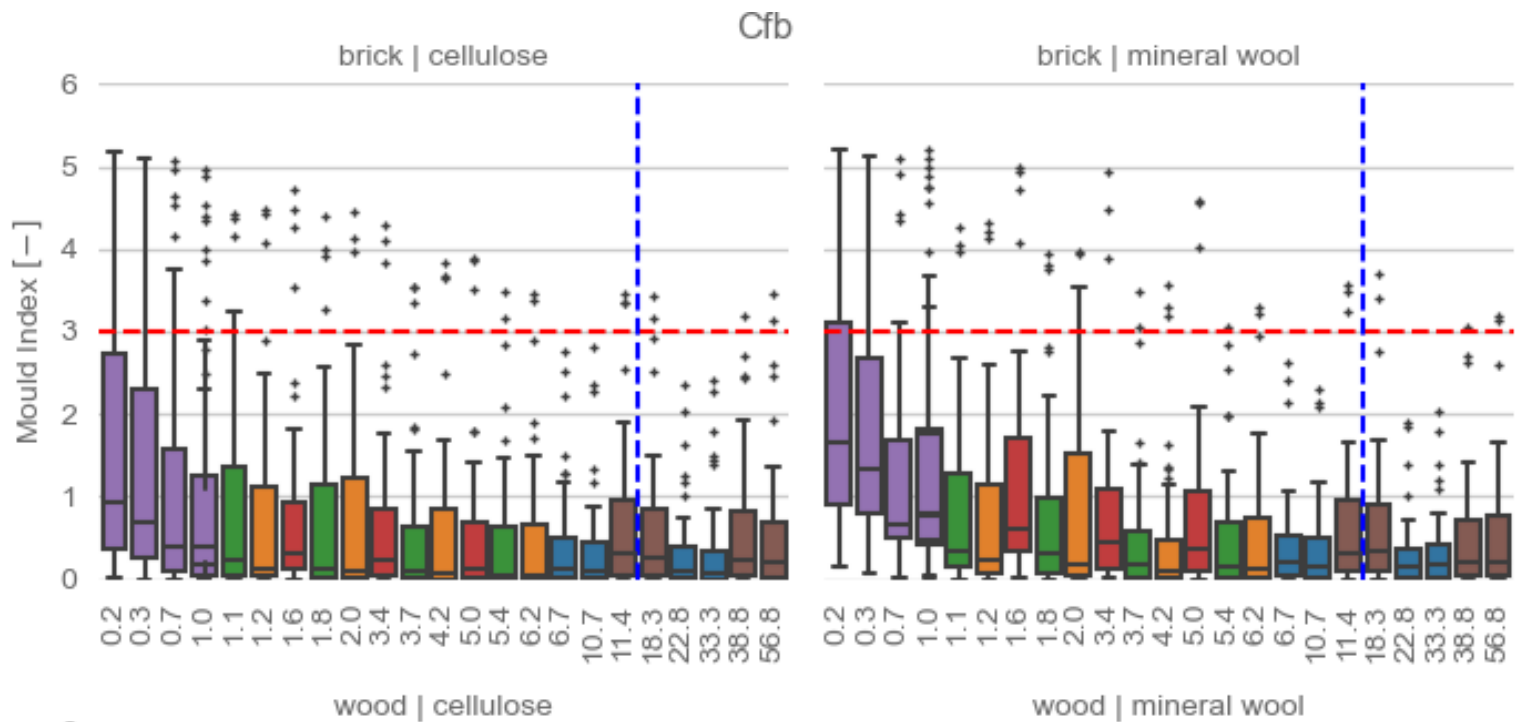

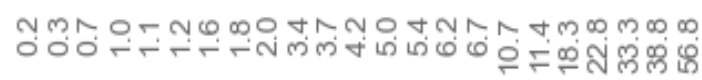
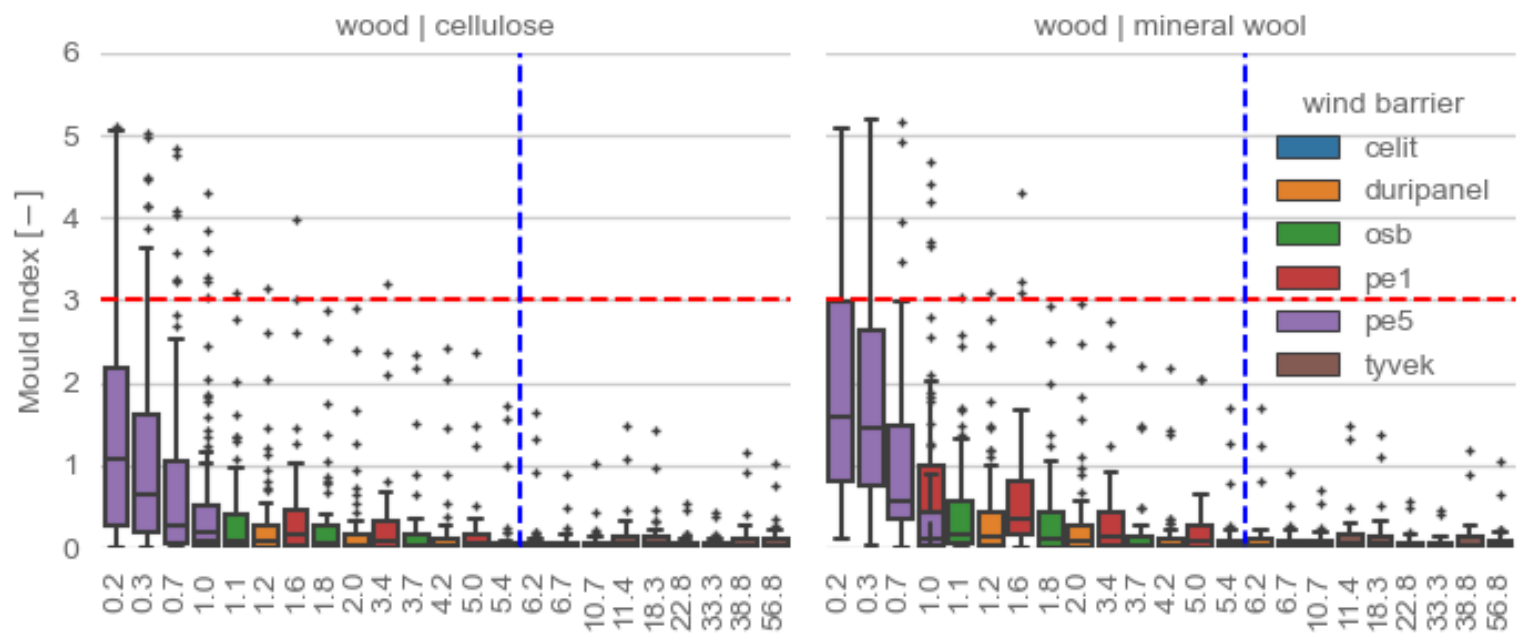

Figure 12: A box plot of the maximum Mould index at the interface between insulation and wind barrier, in function of the $s_{d}$-ratio between the vapour and the wind barrier, for climate class $\mathrm{Cfb}$. The red line indicates the threshold to avoid damage, the blue line indicates the recommended minimum $s_{d}$-ratio as proposed by [2]. Note that the x-axis values are not evenly spaced. 
Postprint: Tijskens A., Roels S., Janssen H., 2019. Hygrothermal assessment of timber frame walls using a convolutional neural network. Building and Environment 193, 107652

https://doi.org/10.1016/j.buildenv.2021.107652

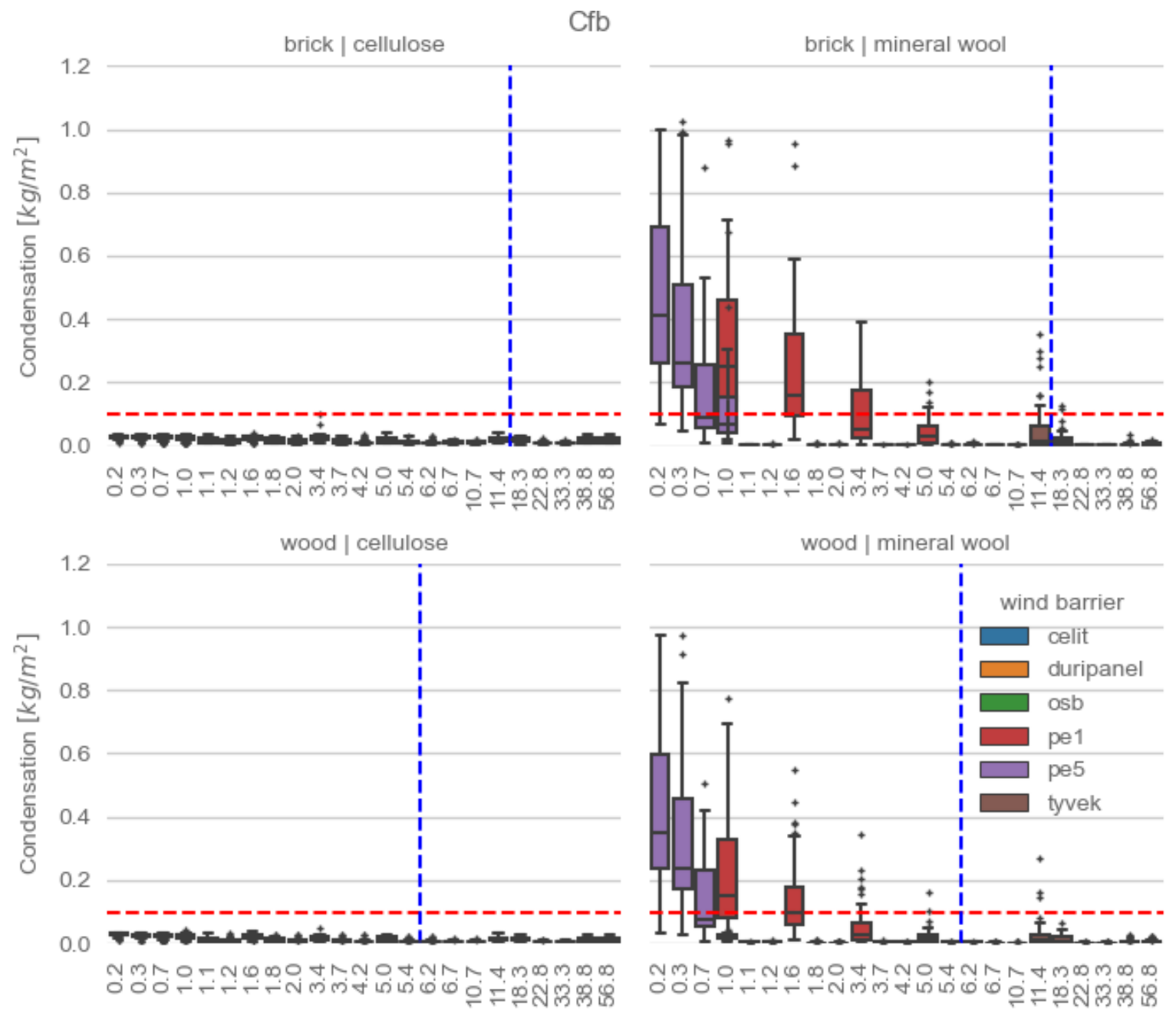

Figure 13: A box plot of the maximum condensation rate at the interface between insulation and wind barrier, in function of the $s_{d}$-ratio between the vapour and the wind barrier, for climate class $\mathrm{Cfb}$. The red line indicates the threshold to avoid damage, the blue line indicates the recommended minimum $s_{d}$-ratio as proposed by [2]. Note that the x-axis values are not evenly spaced.

For climate class Dfc, Figure 14 and Figure 15 show the maximum Mould Index and condensation rate respectively, in function of the $s_{d}$-ratio. For Finland, [3] recommends a ratio of at least 5 for walls with a wooden cladding. As brick cladding is less common in Finland, no recommendations are made for these type of walls. Regarding mould growth risk, this ratio is very conservative and rules out many wind and vapour barrier combinations that would be perfectly safe. The condensation risk shows a different story though. Figure 15 clearly shows high condensation risks for walls with mineral wool insulation and foil wind barriers, even for much higher $s_{d}$-ratios then recommended. On the other 
Postprint: Tijskens A., Roels S., Janssen H., 2019. Hygrothermal assessment of timber frame walls using a convolutional neural network. Building and Environment 193, 107652

https://doi.org/10.1016/j.buildenv.2021.107652

hand, walls with cellulose insulation do not show a condensation risk, nor do walls with mineral wool insulation and a buffering wind barrier.

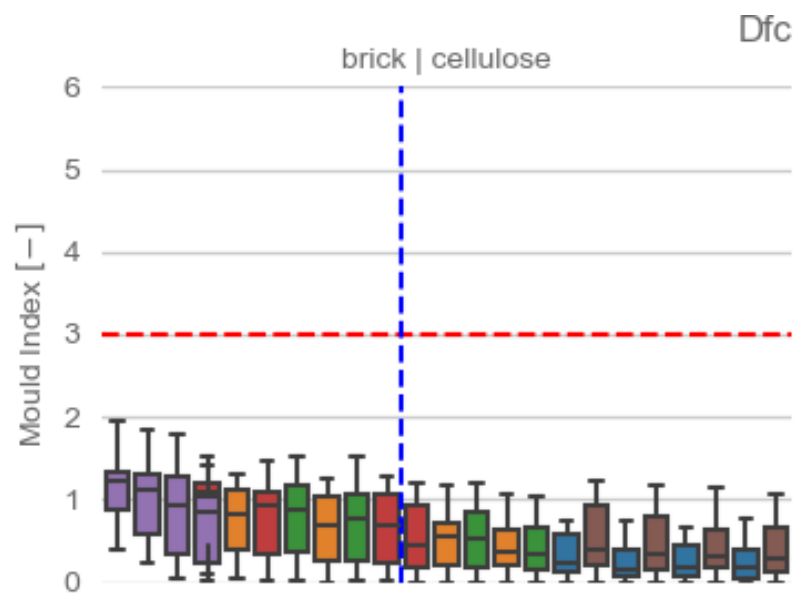

Nmm O० - - N M

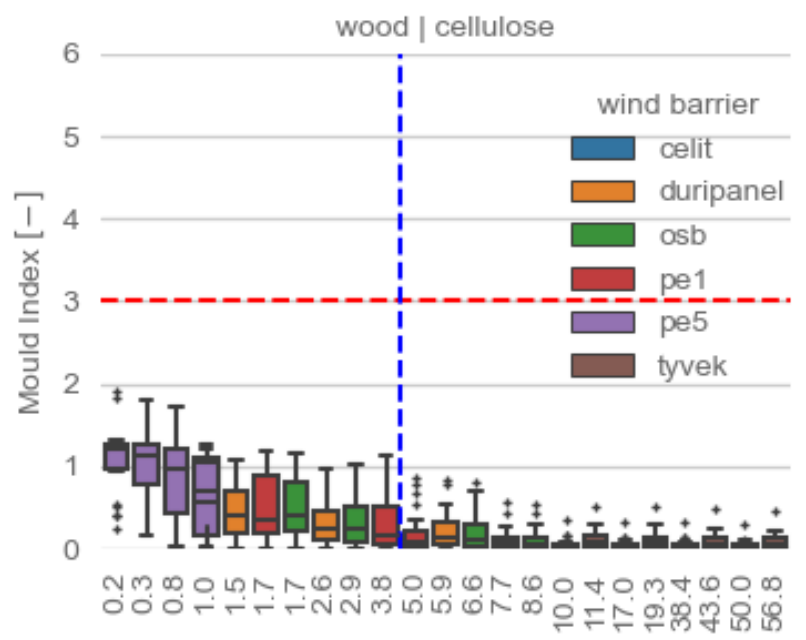

c

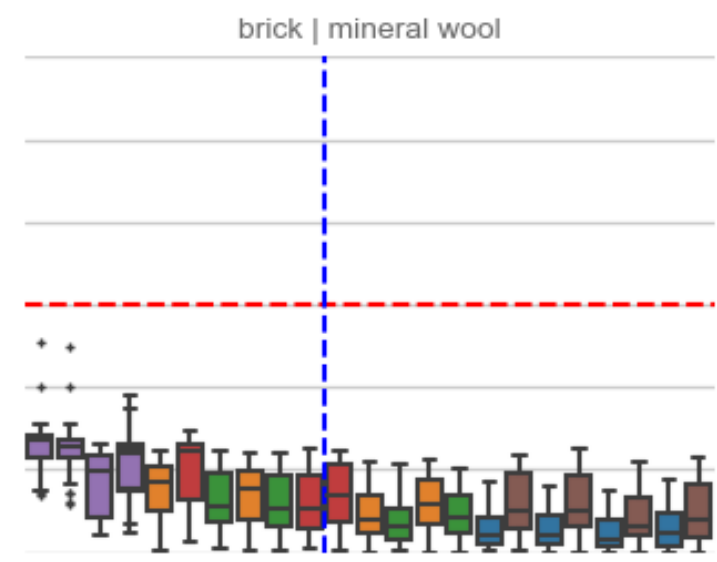

Nm⿻ O० - - N N

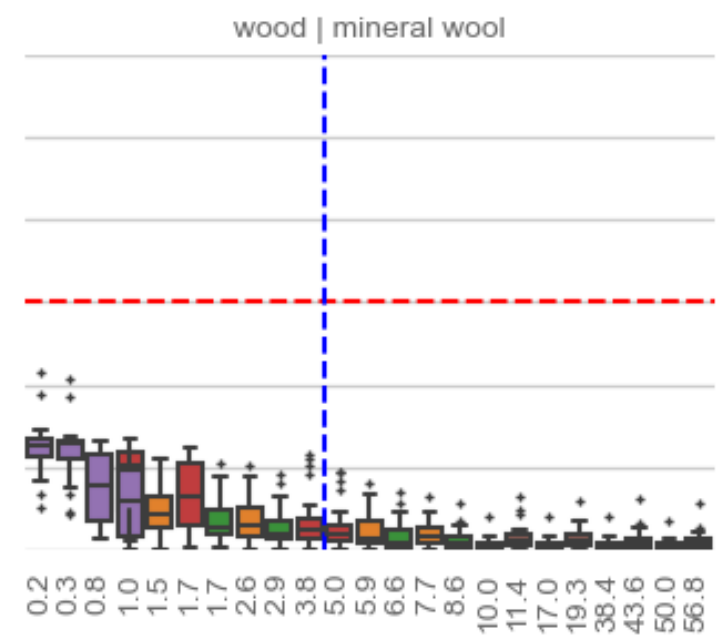

Figure 14: A box plot of the maximum Mould index at the interface between insulation and wind barrier, in function of the $s_{d}$-ratio between the vapour and the wind barrier, for climate class Dfc. The red line indicates the threshold to avoid damage, the blue line indicates the recommended minimum $s_{d}$-ratio as proposed by [3]. Note that the x-axis values are not evenly spaced. 
Postprint: Tijskens A., Roels S., Janssen H., 2019. Hygrothermal assessment of timber frame walls using a convolutional neural network. Building and Environment 193, 107652

https://doi.org/10.1016/j.buildenv.2021.107652
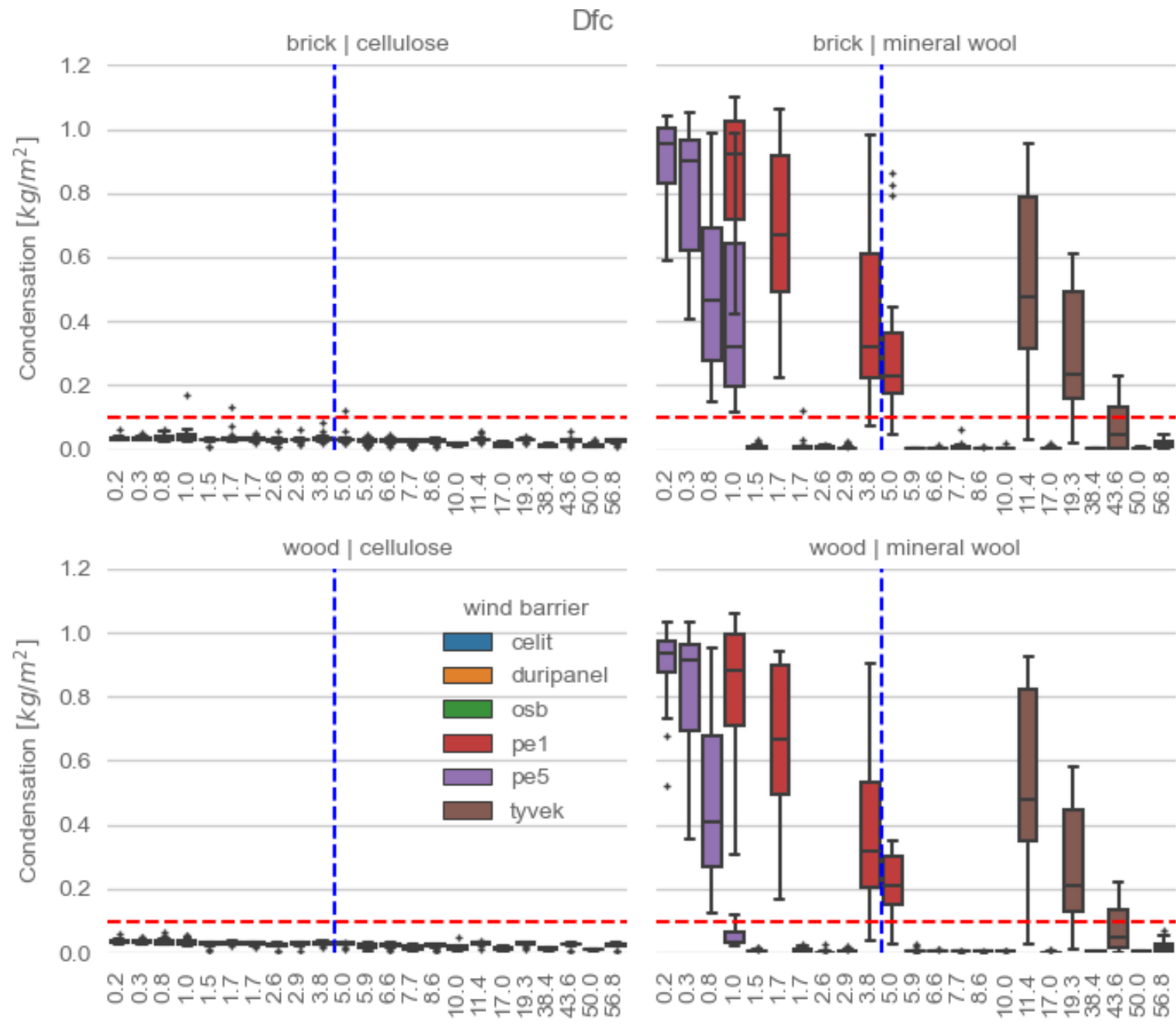

Nm

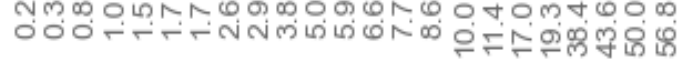

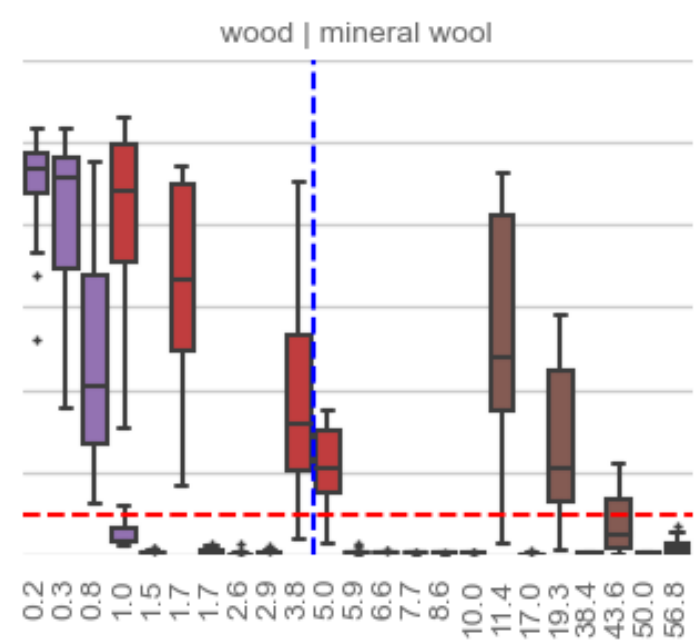

Figure 15: A box plot of the maximum condensation rate at the interface between insulation and wind barrier, in function of the $s_{d}$-ratio between the vapour and the wind barrier, for climate class Dfc. The red line indicates the threshold to avoid damage, the blue line indicates the recommended minimum $s_{d}$-ratio as proposed by [3]. Note that the $\mathrm{x}$-axis values are not evenly spaced.

\section{Conclusion}

In the first part of this paper, a convolutional neural network is used to replace HAM models, aiming to predict the hygrothermal time series response of timber frame walls. A network architecture previously proved accurate for predicting the hygrothermal response of masonry walls is adapted and fine-tuned. The network's hyper -parameters are optimised, using the Grey-Wolf Optimiser algorithm and a limited training dataset. Based on the GWO optimisation of the networks developed for both the massive masonry wall and the lightweight timber frame walls, some general conclusions were drawn: 
Postprint: Tijskens A., Roels S., Janssen H., 2019. Hygrothermal assessment of timber frame walls using a convolutional neural network. Building and Environment 193, 107652

https://doi.org/10.1016/j.buildenv.2021.107652

- The receptive field should be large enough (>11 months) but not (much) larger than the time series length.

- Using fewer layers and stacks reduces the training time. A larger (initial) filter width allows using fewer layers and stacks to obtain the same receptive field.

- Using a low number of filters reduces the training time. To allow complexity, the number of filters should not be to low (64-256).

- A learning rate between 0.001 and 0.0025 is optimal.

As most common wall types are covered between both studies, the above-mentioned guidelines are likely applicable to most wall constructions, and a new hyper-parameters optimisation is not required.

After finishing the GWO algorithm, the 10 best performing hyper-parameter combinations were trained again on a larger dataset, using cross-validation to estimate the generalisation error. The network with the lowest generalisation error was selected and evaluated on an independent test set. These results showed that the developed convolutional neural network is able to capture the complex hygrothermal response of timber frame walls accurately.

In the second part of this study, the selected neural network was used to predict the hygrothermal response of 96 timber frame wall types, taking into account all influencing uncertainties. A multilayered sampling scheme was used to allow reliable comparison of the different wall types, and 128 samples per wall type were simulated. The network needed only 4 minutes to calculate the hygrothermal response of all 12288 samples, which is over 500 times faster compared to the original hygrothermal model. Subsequently, the mould growth and condensation run-off risk were evaluated at the interface between insulation and wind or vapour barrier. The results indicated that timber frame wall compositions should not be recommended based solely on the $s_{d}$-ratio between vapour and wind barrier. A lower limit for the $s_{d}$-ratio appears a good criterion to avoid mould growth, but different climates as well as different cladding types require an adapted guideline. And more important, a lower limit for the $s_{d}$-ratio does not avoid condensation run-off. Instead, one should ensure either the insulation or the wind barrier can buffer the excess moisture. This excludes combining mineral wool insulation with a foil wind barrier. 
Postprint: Tijskens A., Roels S., Janssen H., 2019. Hygrothermal assessment of timber frame walls using a convolutional neural network. Building and Environment 193, 107652

https://doi.org/10.1016/j.buildenv.2021.107652

\section{Acknowledgement}

Funding: This research was funded by the European Union's Horizon 2020 research and innovation program under grant agreement No 637268.

Conflicts of Interest: The authors declare no conflict of interest. The funders had no role in the design of the study; in the collection, analyses, or interpretation of data; in the writing of the manuscript, or in the decision to publish the results.

\section{References}

[1] J. Straube and G. Finch, "Report 0906: Ventilated wall claddings: review, field performance, and hygrothermal modeling," 2009.

[2] "Vochtbeheersing bij houtbouw 2013/01.04," WTCB-Contact nr. 37, Brussel (Belgium), pp. 1215, 2013.

[3] J. Vinha and P. Käkelä, "Water vapour transmissions in wall structures due to diffusion and convection." 1999.

[4] Oak Ridge National Laboratory and A. Karagiozis, Building Enclosure Hygrothermal Performance Study, no. April. 2002.

[5] H. Janssen, "Monte-Carlo based uncertainty analysis: Sampling efficiency and sampling convergence," Reliability Engineering \& System Safety, vol. 109, pp. 123-132, 2013.

[6] A. Tijskens, H. Janssen, and S. Roels, "Optimising Convolutional Neural Networks to Predict the Hygrothermal Performance of Building Components," Energies, vol. 12, no. 20, p. 3966, 2019.

[7] A. Tijskens, S. Roels, and H. Janssen, "Neural networks for metamodelling the hygrothermal behaviour of building components," Building and Environment, vol. 162, no. June, p. 106282, 2019.

[8] A. Tijskens, H. Janssen, and S. Roels, "The impact of a reduced training subspace on the prediction accuracy of neural networks for hygrothermal predictions networks for hygrothermal predictions," Journal of Building Performance Simulation, vol. 14, no. 1, pp. 2037, 2021.

[9] M. Vanpachtenbeke, J. Langmans, J. Van den Bulcke, J. Van Acker, and S. Roels, "Hygrothermal behaviour of timber frame walls finished with a brick veneer cladding," Energy Procedia, vol. 132, pp. 363-368, Oct. 2017.

[10] “Delphin 6.0 [Computer software]." TU Dresden, 2019.

[11] European committee for Standardisation, “EN 15026:2007 - Hygrothermal performance of building components and building elements - Assessment of moisture transfer by numerical simulation," 2007.

[12] European Commission, "Climate for Culture: damage risk assessment, economic impact and mitigation strategies for sustainable preservation of cultural heritage in times of climate change," 2014. 
Postprint: Tijskens A., Roels S., Janssen H., 2019. Hygrothermal assessment of timber frame walls using a convolutional neural network. Building and Environment 193, 107652

https://doi.org/10.1016/j.buildenv.2021.107652

[13] H. E. Beck, N. E. Zimmermann, T. R. McVicar, N. Vergopolan, A. Berg, and E. F. Wood, "Present and future Köppen-Geiger climate classification maps at 1-km resolution," Scientific Data, vol. 5, no. 1, p. 180214, 2018.

[14] International Panel on Climate Change, "IPCC Special Report - Emissions scenarios," 2000.

[15] B. Blocken and J. Carmeliet, "Spatial and temporal distribution of driving rain on a low-rise building," Wind and Structures, An International Journal, vol. 5, no. 5, pp. 441-462, 2002.

[16] J. Langmans and S. Roels, "Experimental analysis of cavity ventilation behind rainscreen cladding systems: A comparison of four measuring techniques," Building and Environment, vol. 87, pp. 177-192, 2015.

[17] M. Vanpachtenbeke, J. Langmans, J. Van den Bulcke, J. Van Acker, and S. Roels, "On the drying potential of cavity ventilation behind brick veneer cladding: A detailed field study," Building and Environment, vol. 123, pp. 133-145, 2017.

[18] ASHRAE, "RP 1091 - Development of Design Strategies for Rainscreen and Sheathing Membrane Performance in Wood Frame Walls," Atlanta, Gorrgia, USA.

[19] A. Janssens, Reliable Control of interstitial condensation in lightweight roof systems. Calculation and assessment methods, vol. phd, no. December. 1998.

[20] J. Langmans, "Feasibility of exterior air barriers in light weight construction," KU Leuven, 2013.

[21] M. Vanpachtenbeke, "Timber frame walls with brick veneer cladding: reliability to fungal decay," KU Leuven, 2019.

[22] T. Ojanen, H. Viitanen, R. Peuhkuri, K. Lähdesmäki, J. Vinha, and K. Salminen, "Mold Growth Modeling of Building Structures Using Sensitivity Classes of Materials," in Thermal Performance of the Exterior Envelopes of Buildings XI, 2010, pp. 1-10.

[23] H. Viitanen, T. Ojanen, and R. Peuhkuri, "Mould growth modelling to evaluate durability of materials," in Proceedings of the 12DBMC - International Conference on Durability of Building Materials and Components, 2011, pp. 1-8.

[24] K. He, X. Zhang, S. Ren, and J. Sun, "Deep Residual Learning for Image Recognition," in IEEE Conference on Computer Vision and Pattern Recognition (CVPR), 2015, vol. 19, no. 2, pp. 107117.

[25] D. P. Kingma and J. Ba, "Adam: A Method for Stochastic Optimization," pp. 1-15, 2014.

[26] S. Mirjalili, S. M. Mirjalili, and A. Lewis, "Grey Wolf Optimizer," Advances in Engineering Software, vol. 69, pp. 46-61, 2014.

[27] L. Van Gelder, H. Janssen, and S. Roels, "Probabilistic design and analysis of building performances: Methodology and application example," Energy and Buildings, vol. 79, pp. 202211, 2014. 